NISSUNA UMANA INVESTIGAZIONE SI PUO DIMANDARE VERA SCIENZIA S'ESSA NON PASSA PER LE MATEMATICHE DIMOSTRAZIONI LEONARDO DA VINCI

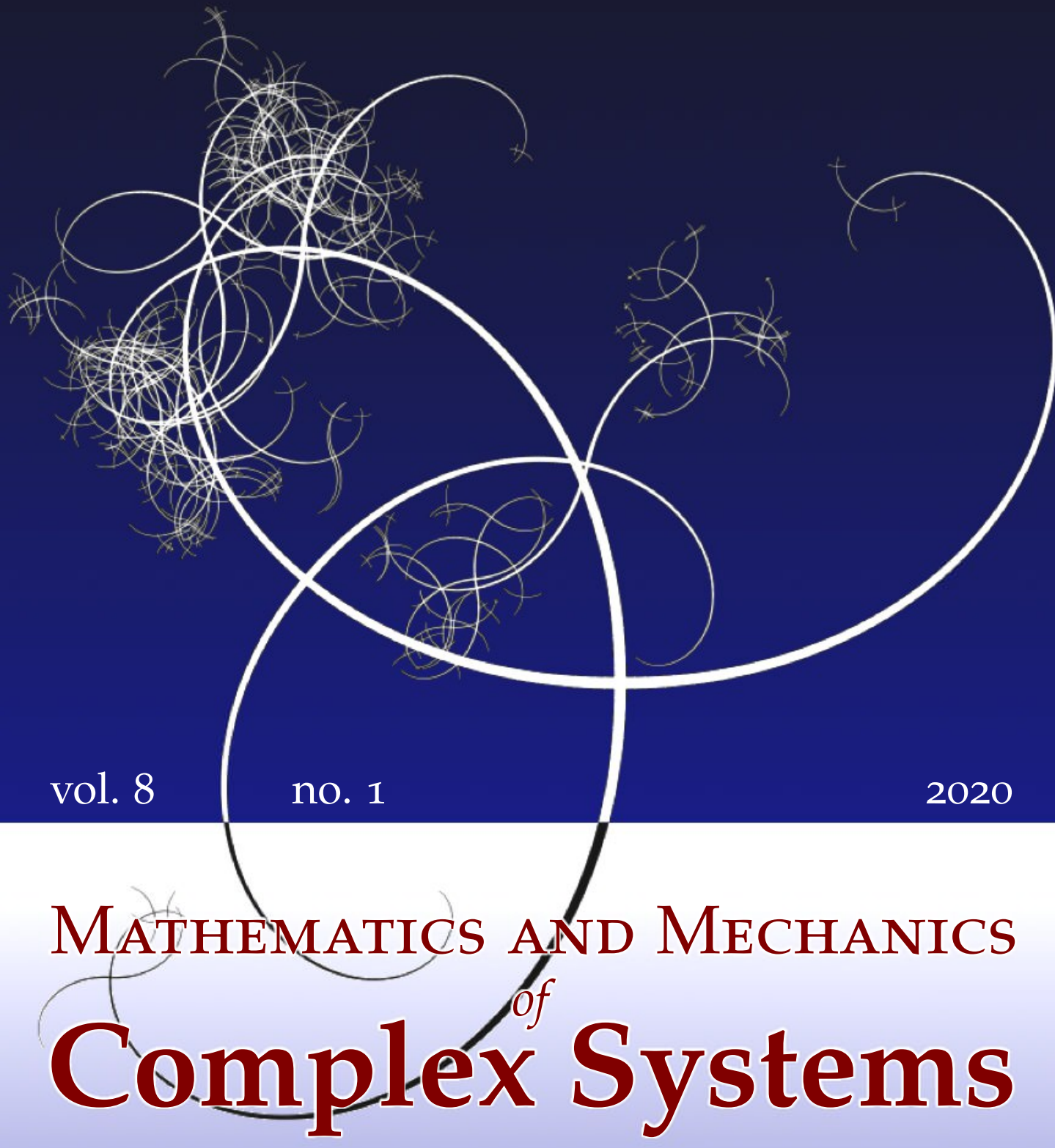

Brett Hendrickson, Milad Shirani and David J. Steigmann EQUILIBRIUM THEORY FOR A LIPID BILAYER WITH A CONFORMING CYTOSKELETAL MEMBRANE 


\title{
EQUILIBRIUM THEORY FOR A LIPID BILAYER WITH A CONFORMING CYTOSKELETAL MEMBRANE
}

\author{
Brett Hendrickson, Milad Shirani and David J. Steigmann
}

We discuss the mechanics of a lipid bilayer with a conforming cytoskeletal membrane in which the bilayer has the structure of a nematic liquid crystal and the cytoskeleton that of a simple elastic solid. Under certain conditions the cytoskeletal membrane mimics the effects of the so-called spontaneous curvature of the conventional theory of lipid membranes. The model is used to predict the classical biconcave discoid shape of red-blood cells in equilibrium.

\section{Introduction}

In this work we outline a model of the elastic response of a lipid bilayer with a conforming cytoskeletal membrane. This is intended for application to the mechanics of red-blood cells, which are known to consist of bilayers with subsurface cytoskeletal membranes formed by spectrin filaments arranged in networks that exhibit 6-fold hexagonal symmetry [Pan et al. 2018]. The basic framework of our model is similar to that underpinning Krishnaswamy's pioneering work [Krishnaswamy 1996] in which material points of the bilayer and cytoskeleton are assumed to be tethered by a so-called connector field while occupying distinct surfaces. The role of this connector is to maintain contact between the bilayer and cytoskeleton as they deform. In that work the bilayer is regarded as a fluid shell, as in Jenkins' model [Jenkins 1977], and the cytoskeleton is considered to be a perfectly flexible solid membrane. Current work on the mechanics of the cytoskeleton [Kamm and Mofrad 2006; Herant and Dembo 2006] suggests that the extent to which it convects with the bilayer is largely unknown. In the present work we therefore take the conservative view that the role of Krishnaswamy's connector is confined to maintaining congruency of the cytoskeletal and bilayer surfaces while playing no significant further role in the mechanical response.

In Section 2 we develop the model of the bilayer/cytoskeleton system via asymptotic expansion in which the bilayer is regarded as a thin nematic liquid crystal film and the cytoskeleton as a thin layer of a simple elastic solid. Certain vector

\section{Communicated by Francesco dell'Isola.}

MSC2010: 53B50, 74K25.

Keywords: lipid bilayers, membrane cytoskeleton, differential geometry. 
fields arising in this procedure occur algebraically in the reduced model and are accordingly evaluated before proceeding further. This is explained in Section 3. In Section 4 we discuss material symmetry conditions for the cytoskeleton and bilayer. Some basic aspects of the differential geometry of surfaces [Naghdi 1972; Ciarlet 2005] are recalled in Section 5 and adapted there to the kinematics of congruent configurations of the bilayer and cytoskeleton. Equilibrium equations are deduced in Section 6 on the basis of a patchwise virtual-power postulate, and restrictions implied by the operative versions of the Legendre-Hadamard condition are discussed in Section 7. We conclude, in Section 8, with a derivation of a strain-energy function for the cytoskeleton which is such as to admit a surface having the shape of the characteristic biconcave discoid of a red-blood cell as an equilibrium state.

\section{Leading-order asymptotic energy for small thickness}

Consider a configuration of the bilayer-cytoskeletal combination in the shape of a prismatic cylinder generated by the parallel translation of a plane region $\Pi$ forming the interface of the bilayer and cytoskeleton (Figure 1). The lipids of the bilayer are presumed to be straight, parallel and of uniform length in this configuration. The bilayer has thickness $\alpha h$ and the cytoskeleton $(1-\alpha) h$, where $h$ is the thickness of the cylinder and $\alpha \in[0,1]$.

The energy of the cylinder is

$$
\mathcal{E}=\int_{\Pi} \mathcal{U} d A
$$

where

$$
\mathcal{U}=\int_{0}^{\alpha h} \mathcal{U}_{b} d \varsigma+\int_{-(1-\alpha) h}^{0} \mathcal{U}_{c} d \varsigma
$$

in which $\mathcal{U}_{b}$ and $\mathcal{U}_{c}$ respectively are the volumetric energy densities of the bilayer and cytoskeleton and $\varsigma$ is a through-thickness coordinate.

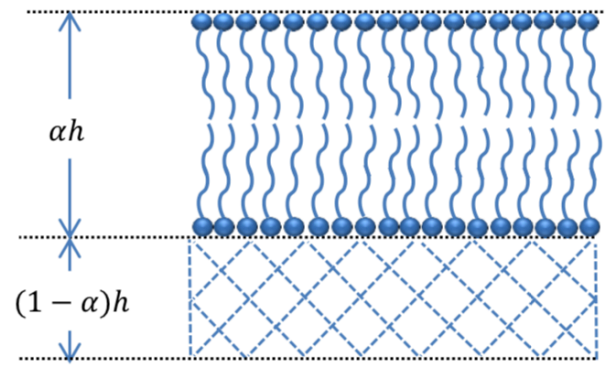

Figure 1. A patch of the bilayer and cytoskeleton. 
A central aspect of the model to be developed is that $\Pi$ is assumed to convect as a material surface with respect to both the bilayer and the cytoskeleton deformations so as to maintain congruency; that is, the (possibly distinct) images of $\Pi$ under the bilayer and cytoskeletal deformations are subsets of a single surface $\omega$. We elaborate on the kinematical implications of this restriction below. Here we assume that $\omega$ can be covered completely by the images of such patches, each of which is assumed, for the sake of notational convenience, to be parametrized by a single coordinate chart.

We suppose the thickness $h$ to be much smaller than any other length scale, $l$ say, in a given problem. If the latter is used as the unit of length $(l=1)$, then the dimensionless thickness $h \ll 1$. Regarding $\mathcal{U}$ as a function of $h$, we combine Leibniz's rule with a Taylor expansion to derive

$$
\mathcal{U}=h U+o(h), \quad \text { with } \quad U=\alpha U_{b}+(1-\alpha) U_{c},
$$

in which $U_{b}$ and $U_{c}$ respectively are the values of $\mathcal{U}_{b}$ and $\mathcal{U}_{c}$ at $\varsigma=0$, i.e., at their common interface $\Pi$. Accordingly,

$$
\mathcal{E} / h=E+o(h) / h, \quad \text { where } \quad E=\int_{\Pi} U d A,
$$

is the leading-order energy for small $h$.

Alternatively, in view of the fact that the thickness of the bilayer/cytoskeleton composite is on the order of molecular dimensions, it is appropriate to contemplate a direct theory based at the outset on the idea of a material surface without regard to thickness effects. However, the present asymptotic approach offers guidance as to the features that such a direct model should possess.

We assume the cytoskeleton to be a uniform elastic material with a strain energy given by

$$
\mathcal{U}_{c}=\mathcal{W}_{c}(\widetilde{\boldsymbol{F}})
$$

where $\widetilde{\boldsymbol{F}}$ is the gradient of the cytoskeletal deformation $\tilde{\chi}(\boldsymbol{x})$, with $\boldsymbol{x} \in \Pi \times[-(1-$ $\alpha) h, 0]$, i.e., $\boldsymbol{x}=\boldsymbol{\xi}+\varsigma \boldsymbol{k}$, where $\boldsymbol{\xi}$ is the projection of $\boldsymbol{x}$ onto the plane region $\Pi$ with unit normal $\boldsymbol{k}$ and $\varsigma \in[-(1-\alpha) h, 0]$. Thus, $\widetilde{\boldsymbol{F}}=\hat{\boldsymbol{F}}(\boldsymbol{\xi}, \varsigma)$, where

$$
\hat{\boldsymbol{F}}=\nabla \hat{\chi}+\hat{\chi}^{\prime} \otimes \boldsymbol{k} .
$$

Here $(\cdot)^{\prime}=\partial(\cdot) / \partial \varsigma, \nabla(\cdot)$ is the (two-dimensional) gradient with respect to $\xi$, and $\hat{\chi}(\xi, \varsigma)=\tilde{\chi}(\xi+\varsigma k)$. Then,

$$
U_{c}=\mathcal{W}_{c}(\boldsymbol{F}), \quad \text { where } \quad \boldsymbol{F}=\nabla \boldsymbol{r}_{c}+\boldsymbol{d} \otimes \boldsymbol{k},
$$

is the restriction to $\Pi$ of the cytoskeletal deformation gradient, in which $\boldsymbol{r}_{c}(\boldsymbol{\xi})=\hat{\chi}_{\mid \Pi}$ is the interfacial cytoskeletal deformation and $\boldsymbol{d}(\boldsymbol{\xi})=\hat{\chi}_{\mid \Pi}^{\prime}$ is the interfacial value of the normal derivative of the deformation. 
Following Helfrich [1973], we model the lipid bilayer as a liquid crystal with an energy density

$$
\mathcal{U}_{b}=\mathcal{W}_{b}(\tilde{\boldsymbol{n}}, \widetilde{\boldsymbol{D}})
$$

where $\tilde{\boldsymbol{n}}$ is a field of unit vectors specifying the local molecular orientation and $\widetilde{\boldsymbol{D}}=\operatorname{grad} \tilde{\boldsymbol{n}}$ is its (spatial) gradient. It is customary [Virga 1994] to specify a constitutive function for the energy per unit current volume and to regard the liquid crystal as an incompressible medium. Accordingly $\mathcal{U}_{b}$ is also the energy per unit reference volume, as assumed in the foregoing. Then,

$$
U_{b}=\mathcal{W}_{b}(\boldsymbol{n}, \boldsymbol{D}),
$$

where $\boldsymbol{n}$ and $\boldsymbol{D}$ are the interfacial values of $\tilde{\boldsymbol{n}}$ and $\widetilde{\boldsymbol{D}}$, respectively. Here, as in Helfrich's theory [Helfrich 1973], we suppress lipid tilt and thus take $\boldsymbol{n}$ to be the unit-normal field to the image $\pi_{b}$ of the interface $\Pi$ in the current configuration of the lipid/cytoskeleton system. In these circumstances, we have

$$
\boldsymbol{D}=\nabla_{s} \boldsymbol{n}+\boldsymbol{\eta} \otimes \boldsymbol{n},
$$

where $\nabla_{s}(\cdot)$ is the surfacial gradient on $\pi_{b}$ and $\eta$ is the restriction to $\pi_{b}$ of the derivative of $\tilde{\boldsymbol{n}}$ in the direction of $\tilde{\boldsymbol{n}}$. Because the latter is a field of unit vectors, we require $\boldsymbol{n} \cdot \boldsymbol{\eta}=0$ and conclude that $\boldsymbol{\eta}$ is a tangential vector field on $\pi_{b}$.

The Gauss and Weingarten equations of differential geometry furnish

$$
\nabla_{s} \boldsymbol{n}=-\boldsymbol{b},
$$

where $\boldsymbol{b}$ is the symmetric curvature 2-tensor on the local tangent planes of $\pi_{b}$. We elaborate further in Section 5 below.

The energy density of the composite is thus given, in an abuse of notation, by the function

$$
U\left(\nabla \boldsymbol{r}_{c}, \boldsymbol{b}, \boldsymbol{d}, \boldsymbol{n}, \boldsymbol{\eta}\right)=\alpha U_{b}(\boldsymbol{b}, \boldsymbol{n}, \boldsymbol{\eta})+(1-\alpha) U_{c}\left(\nabla \boldsymbol{r}_{c}, \boldsymbol{d}\right),
$$

where

$$
U_{b}(\boldsymbol{b}, \boldsymbol{n}, \boldsymbol{\eta})=\mathcal{W}_{b}(\boldsymbol{n},-\boldsymbol{b}+\boldsymbol{\eta} \otimes \boldsymbol{n}) \quad \text { and } \quad U_{c}\left(\nabla \boldsymbol{r}_{c}, \boldsymbol{d}\right)=\mathcal{W}_{c}\left(\nabla \boldsymbol{r}_{c}+\boldsymbol{d} \otimes \boldsymbol{k}\right) .
$$

We observe that the dependence of the energy on the fields $\boldsymbol{d}$ and $\boldsymbol{\eta}$ is purely algebraic. This suggests a strategy, pursued in the next section, whereby we attempt to render the energy stationary with respect to these fields a priori.

\section{Determination of $d$ and $\eta$}

3.1. Cytoskeletal deformation. We decompose $\boldsymbol{d}$ into normal and tangential parts as

$$
\boldsymbol{d}=d_{n} \boldsymbol{n}+\left(\nabla \boldsymbol{r}_{c}\right) \boldsymbol{e}
$$


where $d_{n}=\boldsymbol{d} \cdot \boldsymbol{n}, \boldsymbol{e}$ is a 2-vector on $\Pi$ and $J_{c} \boldsymbol{n}=\boldsymbol{F}^{*} \boldsymbol{k}$, in which $\boldsymbol{F}^{*}$ is the cofactor of $\boldsymbol{F}$, and we note that $\nabla \boldsymbol{r}_{c}$ maps $\Pi$ to the tangent plane of the image $\pi_{c}$ of $\Pi$ under the deformation at the material point in question. Here $J_{c}\left(=\left|\boldsymbol{F}^{*} \boldsymbol{k}\right|\right)$ and $\boldsymbol{n}$ respectively are the areal stretch of the interface due to the deformation of the cytoskeleton and the unit normal to $\pi_{c}$; these are determined by $\nabla \boldsymbol{r}_{c}$. We then have $\operatorname{det} \boldsymbol{F}=\boldsymbol{F} \boldsymbol{k} \cdot \boldsymbol{F}^{*} \boldsymbol{k}=J_{c} d_{n}$ and thus require $d_{n}>0$.

The cytoskeletal energy is frame-invariant if and only if it depends on $\boldsymbol{F}$ via the Cauchy-Green tensor $\boldsymbol{C}=\boldsymbol{F}^{t} \boldsymbol{F}$; we write $\mathcal{W}_{c}(\boldsymbol{F})=F(\boldsymbol{C})$, where, from (7) and (14),

$$
\boldsymbol{C}=\boldsymbol{c}+\boldsymbol{\gamma} \otimes \boldsymbol{k}+\boldsymbol{k} \otimes \boldsymbol{\gamma}+\left(d_{n}^{2}+\boldsymbol{e} \cdot \boldsymbol{c e}\right) \boldsymbol{k} \otimes \boldsymbol{k},
$$

with

$$
\boldsymbol{c}=\left(\nabla \boldsymbol{r}_{c}\right)^{t} \nabla \boldsymbol{r}_{c} \quad \text { and } \quad \boldsymbol{\gamma}=\boldsymbol{c e},
$$

and we remark that

$$
J_{c}^{2}=\operatorname{det} \boldsymbol{c} .
$$

Let $G(\boldsymbol{e})=F(\boldsymbol{C}(\boldsymbol{e}))$, where $\boldsymbol{C}(\boldsymbol{e})$ is the function obtained by fixing $d_{n}$ and $\nabla \boldsymbol{r}_{c}$ in (15). We seek 2-vectors $\boldsymbol{e}$ that render $G$ stationary. Consider materials that exhibit reflection symmetry with respect to the plane $\Pi$, i.e., $F(\boldsymbol{C})=F\left(\boldsymbol{R}^{t} \boldsymbol{C} \boldsymbol{R}\right)$ with $\boldsymbol{R}=\boldsymbol{I}-2 \boldsymbol{k} \otimes \boldsymbol{k}$, in which $\boldsymbol{I}$ is the three-dimensional identity. Thus,

$$
\boldsymbol{R}^{t} \boldsymbol{C} \boldsymbol{R}=\boldsymbol{c}-\boldsymbol{\gamma} \otimes \boldsymbol{k}-\boldsymbol{k} \otimes \boldsymbol{\gamma}+\left(d_{n}^{2}+\boldsymbol{e} \cdot \boldsymbol{c e}\right) \boldsymbol{k} \otimes \boldsymbol{k},
$$

and so reflection symmetry implies that $G$ is an even function: $G(\boldsymbol{e})=G(-\boldsymbol{e})$. It follows that there is a function $S$ such that $G(\boldsymbol{e})=S(\boldsymbol{E})$, where $\boldsymbol{E}=\boldsymbol{e} \otimes \boldsymbol{e}$ (see the Appendix). Accordingly, $G_{\boldsymbol{e}}=2\left(S_{\boldsymbol{E}}\right) \boldsymbol{e}$ and the stationarity condition is satisfied if $\boldsymbol{e}=\mathbf{0}$; equation (15) then reduces to

$$
\boldsymbol{C}=\boldsymbol{c}+d_{n}^{2} \boldsymbol{k} \otimes \boldsymbol{k},
$$

and the cytoskeletal energy is determined by $c$ and $d_{n}$ :

$$
U_{c}=F\left(\boldsymbol{c}+d_{n}^{2} \boldsymbol{k} \otimes \boldsymbol{k}\right) .
$$

This is stationary with respect to $d_{n}(>0)$ if and only if

$$
\boldsymbol{k} \cdot\left(F_{C}\right) \boldsymbol{k}=0,
$$

which fixes $d_{n}$ in terms of $\boldsymbol{c}$.

As we are concerned with equilibria, it is appropriate to confine attention to deformations $\boldsymbol{F}$ that satisfy the strong-ellipticity condition; that is, to deformations satisfying

$$
\boldsymbol{a} \otimes \boldsymbol{b} \cdot\left(\mathcal{W}_{c}\right)_{\boldsymbol{F} \boldsymbol{F}}[\boldsymbol{a} \otimes \boldsymbol{b}]>0,
$$


for all $\boldsymbol{a} \otimes \boldsymbol{b} \neq \mathbf{0}$. In these circumstances the stationarity conditions have unique solutions that minimize the energy absolutely [Steigmann 2010].

3.2. The lipid bilayer. We model the lipid bilayer as a nematic liquid crystal described by Frank's energy (see [Virga 1994, (3.63)])

$\mathcal{W}_{b}(\boldsymbol{n}, \boldsymbol{D})=k_{1}(\operatorname{tr} \boldsymbol{D})^{2}+k_{2}(\boldsymbol{W}(\boldsymbol{n}) \cdot \boldsymbol{D})^{2}+k_{3}|\boldsymbol{D} \boldsymbol{n}|^{2}+\left(k_{2}+k_{4}\right)\left[\operatorname{tr}\left(\boldsymbol{D}^{2}\right)-(\operatorname{tr} \boldsymbol{D})^{2}\right]$,

where $k_{1}-k_{4}$ are constants satisfying Ericksen's inequalities

$$
2 k_{1} \geq k_{2}+k_{4}, \quad k_{2} \geq\left|k_{4}\right| \quad \text { and } \quad k_{3} \geq 0,
$$

in accordance with the assumed positive semidefiniteness of $\mathcal{W}_{b}(\boldsymbol{n}, \cdot)$, and $\boldsymbol{W}(\boldsymbol{n})$ is the skew tensor with axial vector $\boldsymbol{n}$, i.e., $\boldsymbol{W}(\boldsymbol{n}) \boldsymbol{v}=\boldsymbol{n} \times \boldsymbol{v}$ for all $\boldsymbol{v}$. Then, with (10) and (11), we have

$$
\boldsymbol{W}(\boldsymbol{n}) \cdot \boldsymbol{D}=\boldsymbol{\eta} \cdot \boldsymbol{W}(\boldsymbol{n}) \boldsymbol{n}-\boldsymbol{W}(\boldsymbol{n}) \cdot \boldsymbol{b}=0,
$$

on account of the symmetry of $\boldsymbol{b}$.

Further,

$$
\operatorname{tr} \boldsymbol{D}=-2 H, \quad \text { where } \quad H=\frac{1}{2} \operatorname{tr} \boldsymbol{b},
$$

is the mean curvature of $\pi_{b}$. Combining

$$
D^{2}=b^{2}-b \eta \otimes n,
$$

with the Cayley-Hamilton formula

$$
\boldsymbol{b}^{2}=2 H \boldsymbol{b}-K \mathbf{1}, \quad \text { where } K=\operatorname{det} \boldsymbol{b},
$$

is the Gaussian curvature of $\pi_{b}$ and $\mathbf{1}=\boldsymbol{I}-\boldsymbol{n} \otimes \boldsymbol{n}$ is the (two-dimensional) identity on its local tangent plane, we arrive at

$$
\operatorname{tr}\left(\boldsymbol{D}^{2}\right)=\operatorname{tr}\left(\boldsymbol{b}^{2}\right)=4 H^{2}-2 K .
$$

Lastly, $\boldsymbol{D} \boldsymbol{n}=\boldsymbol{\eta}$ so that, altogether,

$$
\mathcal{W}_{b}(\boldsymbol{n}, \boldsymbol{D})=k H^{2}+\bar{k} K+k_{3}|\eta|^{2},
$$

with

$$
k=4 k_{1} \quad \text { and } \quad \bar{k}=-2\left(k_{2}+k_{4}\right) .
$$

For $k_{3}$ nonzero this is stationary with respect to $\boldsymbol{\eta}$ at $\boldsymbol{\eta}=\mathbf{0}$, and so we recover the classical Canham-Helfrich energy [Helfrich 1973; Canham 1970]

$$
U_{b}=k H^{2}+\bar{k} K,
$$

for lipid bilayers, which of course covers the possibility that $k_{3}$ vanishes. For $k_{3}>0$, it is clear that (32) furnishes the minimum of (30). 
It is well known that the term in square brackets in (23) is a null Lagrangian in three-dimensional liquid-crystal theory [Virga 1994]. This term is proportional to $K$, a null Lagrangian in the two-dimensional theory of lipid bilayers. Moreover, in this theory it is customary to model a possible asymmetry in bending response by introducing a variable $C$, the spontaneous curvature, via the modified energy [Ou-Yang et al. 1999]

$$
U_{b}=k(H-C)^{2}+\bar{k} K .
$$

There are a number of physical effects that can give rise to a spontaneous curvature. Examples include diffusion of transmembrane proteins [Agrawal and Steigmann 2011] and flexoelectricity [Ou-Yang et al. 1999]. One of our objectives in this work is to demonstrate that a conforming cytoskeletal membrane effectively mimics a spontaneous curvature under certain conditions.

With reference to (3) and (4), the net leading-order composite energy is

$$
E=\int_{\Pi} W d A
$$

where

$$
W=W_{b}(H, K)+W_{c}(\boldsymbol{c}),
$$

with

$$
W_{b}(H, K)=\kappa H^{2}+\bar{\kappa} K \quad \text { and } \quad W_{c}(\boldsymbol{c})=(1-\alpha) F\left(\boldsymbol{c}+d_{n}^{2}(\boldsymbol{c}) \boldsymbol{k} \otimes \boldsymbol{k}\right),
$$

and with $\kappa=\alpha k$ and $\bar{\kappa}=\alpha \bar{k}$.

We adopt the conventional assumption [Evans and Skalak 1980] that deformations of the bilayer/cytoskeleton system conserve local surface area. This assumption is invoked for both the bilayer and cytoskeleton separately. For bilayers it is justified by bulk incompressibility in the parent theory of liquid crystals and by the suppression of lipid tilt. The presumed inextensibility of the lipids - expressed by the condition $|\boldsymbol{n}|=1$ - then implies areal incompressibility. For the cytoskeleton it is justified by empirical evidence [Evans and Skalak 1980] indicating that areal compressibility of the bilayer/cytoskeleton system is typically negligible; areal incompressibility, in the case of a convecting cytoskeleton, then follows from that of the bilayer. Here we impose areal incompressibility of the cytoskeleton whether or not it convects with the bilayer (for a discussion of this issue, see [Krishnaswamy 1996]). Accordingly, the referential areal energy density $W$ is also the areal density in the current configuration of the system in the sense that

$$
E=\int_{\pi_{b}} W_{b} d a+\int_{\pi_{c}} W_{c} d a,
$$

where $\pi_{b} \subset \omega$ and $\pi_{c} \subset \omega$ respectively are the images of $\Pi$ under the bilayer and cytoskeletal deformations. 


\section{Material symmetry}

4.1. The cytoskeleton. Little if anything is known about the symmetry group for the cytoskeleton, regarded as a three-dimensional continuum. However, on the basis of work reported in [Pan et al. 2018] we assume that the two-dimensional response of the cytoskeletal membrane exhibits hexatropic symmetry relative to the plane configuration $\Pi$, characterized by mechanically equivalent unit vectors $\boldsymbol{i}_{1}, \boldsymbol{i}_{2}$ and $\boldsymbol{i}_{3}$ aligned with the filaments of the cytoskeleton (Figure 2).

Thus the function $W_{c}(\boldsymbol{c})$ is assumed to be such that [Cohen and Wang 1984]

$$
W_{c}(\boldsymbol{c})=W_{c}\left(\boldsymbol{R}^{t} \boldsymbol{c} \boldsymbol{R}\right),
$$

for all two-dimensional orthogonal $\boldsymbol{R}$ belonging to the hexatropic symmetry group. This group is characterized in [Zheng et al. 1992], where it is proved that the list $\left\{\operatorname{tr} \boldsymbol{c}, \operatorname{tr}\left(\boldsymbol{c}^{2}\right), \operatorname{tr}\left(\boldsymbol{h}_{c} \boldsymbol{c}\right)\right\}$ is a function basis for hexatropic symmetry [Zheng et al. 1992, Table 1], with

$$
\boldsymbol{h}_{c}=\left[(\boldsymbol{m} \cdot \boldsymbol{c})^{2}-\left(\boldsymbol{m}^{\prime} \cdot \boldsymbol{c}\right)^{2}\right] \boldsymbol{m}-2(\boldsymbol{m} \cdot \boldsymbol{c})\left(\boldsymbol{m}^{\prime} \cdot \boldsymbol{c}\right) \boldsymbol{m}^{\prime},
$$

in which the interposed dot is the inner product on the translation space $\Pi^{\prime}$ of $\Pi$, and

$$
\boldsymbol{m}=\boldsymbol{e}_{1} \otimes \boldsymbol{e}_{1}-\boldsymbol{e}_{2} \otimes \boldsymbol{e}_{2}, \quad \boldsymbol{m}^{\prime}=\boldsymbol{e}_{1} \otimes \boldsymbol{e}_{2}+\boldsymbol{e}_{2} \otimes \boldsymbol{e}_{1},
$$

with

$$
\boldsymbol{e}_{1}=\boldsymbol{i}_{1} \quad \text { and } \quad \boldsymbol{e}_{2}=\left(\boldsymbol{i}_{2}-\boldsymbol{i}_{3}\right) / \sqrt{3}
$$

Alternatively, the Cayley-Hamilton formula yields the equivalent function basis $\left\{\operatorname{tr} \boldsymbol{c}, J_{c}, \operatorname{tr}\left(\boldsymbol{h}_{c} \boldsymbol{c}\right)\right\}$ in which $J_{c}=1$ by virtue of areal incompressibility. We suppress a possible explicit dependence of the strain energy on the material point $\xi \in \Pi$ due to any nonuniformity of the material properties or of the orientation of the triad $\left\{\boldsymbol{i}_{k}\right\}$.

According to prevailing opinion [Evans and Skalak 1980; Tartibi et al. 2015], the cytoskeletal membrane exhibits response that is characteristic of an isotropic

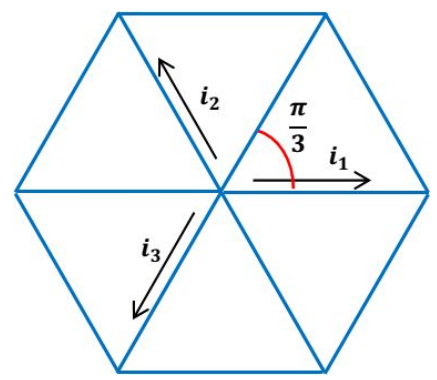

Figure 2. Hexagonal substructure of the cytoskeletal network. 
material. This view must be qualified by the membrane-theoretic version of Noll's rule giving the symmetry group relative to any configuration when that relative to one of them is known, i.e., the membrane, if isotropic relative to one configuration, cannot be isotropic relative to all. Here, to avoid ambiguity, we interpret prevailing opinion as implying isotropy relative to $\Pi$ and thus do not include $\operatorname{tr}\left(\boldsymbol{h}_{c} \boldsymbol{c}\right)$ among the arguments of the strain-energy function. Thus we assume

$$
W_{c}(\boldsymbol{c})=\varpi(I), \quad \text { where } \quad I=\operatorname{tr} c,
$$

for some function $\varpi(\cdot)$. Naturally, the symmetry group is thereby enlarged to the orthogonal group. However, hexatropy may be reconciled with isotropy if the strain $\boldsymbol{\epsilon}$, defined by $2 \boldsymbol{\epsilon}=\boldsymbol{c}-\mathbf{1}_{\Pi}$, where $\mathbf{1}_{\Pi}$ is the identity on $\Pi^{\prime}$, is sufficiently small.

Hexatropy implies that the strain energy, expressed as a function of the strain, has as arguments the elements of the function basis $\left\{\operatorname{tr} \boldsymbol{\epsilon}, \operatorname{tr}\left(\boldsymbol{\epsilon}^{2}\right), \operatorname{tr}\left(\boldsymbol{h}_{\epsilon} \boldsymbol{\epsilon}\right)\right\}$, where $\boldsymbol{h}_{\epsilon}$ is defined by (39) with $\boldsymbol{c}$ replaced by $\boldsymbol{\epsilon}$. This function basis is approximated at quadratic order in $\epsilon$ by the basis $\left\{\operatorname{tr} \epsilon, \operatorname{tr}\left(\epsilon^{2}\right)\right\}$ for isotropy. Thus, the view expressed in the literature is consistent with the substructure of the cytoskeletal network if terms through quadratic order in $\epsilon$ are retained in the strain-energy function. Indeed, quadratic-order energies figure prominently in Evans' and Skalak's extensive treatment [Evans and Skalak 1980] of cytoskeletal membranes in which isotropy is assumed at the outset.

4.2. The bilayer. The bilayer energy may also be interpreted in the framework of material symmetry. It is known, in the case of areal incompressibility [Steigmann 2003; Zheng 2003], that any function of the mean and Gaussian curvatures $H$ and $K$ may be expressed as a function, $B$ say, of $\boldsymbol{c}=(\nabla \boldsymbol{r})^{t}(\nabla \boldsymbol{r})$ and the bending strain $\boldsymbol{\kappa}=(\nabla \boldsymbol{r})^{t} \boldsymbol{b}(\nabla \boldsymbol{r})$, where $\boldsymbol{r}(\boldsymbol{\xi})$ is the bilayer deformation, provided that

$$
B(\boldsymbol{c}, \boldsymbol{\kappa})=B\left(\boldsymbol{R}^{t} \boldsymbol{c} \boldsymbol{R}, \pm \boldsymbol{R}^{t} \kappa \boldsymbol{R}\right),
$$

for all two-dimensional unimodular $\boldsymbol{R}(|\operatorname{det} \boldsymbol{R}|=1)$, with the sign chosen in accordance with that of det $\boldsymbol{R}$. Here the minus sign is associated with the reflection symmetry of bilayers. This restriction has its origins in Murdoch's and Cohen's extension [Murdoch and Cohen 1979] of Noll's concept [Noll 1958] of material symmetry to elastic surfaces, and comports with his use of the concept of material symmetry [Noll 2004] in the interpretation of the constitutive response of liquid crystals.

\section{Surface differential geometry}

A configuration of the bilayer/cytosleletal system occupies a surface $\omega$, which we parametrize as $\boldsymbol{r}\left(\theta^{\alpha}\right)$ in which $\theta^{\alpha}, \alpha=1,2$, are surface coordinates. The surface 
parametrization induces the tangent basis $\left\{\boldsymbol{a}_{\alpha}\right\}$, where $\boldsymbol{a}_{\alpha}=\boldsymbol{r}_{, \alpha}$; the (invertible) surface metric $a_{\alpha \beta}=\boldsymbol{a}_{\alpha} \cdot \boldsymbol{a}_{\beta}$; the dual metric $a^{\alpha \beta}$, where $\left(a^{\alpha \beta}\right)=\left(a_{\alpha \beta}\right)^{-1}$; and the dual tangent basis $\left\{\boldsymbol{a}^{\alpha}\right\}$, with $\boldsymbol{a}^{\alpha}=a^{\alpha \beta} \boldsymbol{a}_{\beta}$. The orientation of $\omega$ is specified by the unit-normal field $\boldsymbol{n}$ defined by $\varepsilon_{\alpha \beta} \boldsymbol{n}=\boldsymbol{a}_{\alpha} \times \boldsymbol{a}_{\beta}$, where $\varepsilon_{\alpha \beta}=\sqrt{a} e_{\alpha \beta}$, with $a=\operatorname{det}\left(a_{\alpha \beta}\right)$, is the Levi-Civita alternating tensor and $e_{\alpha \beta}$ the permutation symbol $\left(e_{12}=-e_{21}=1, e_{11}=e_{22}=0\right)$.

Central to our development are the Gauss and Weingarten equations [Ciarlet 2005; Naghdi 1972]

$$
\boldsymbol{r}_{; \alpha \beta}=b_{\alpha \beta} \boldsymbol{n} \quad \text { and } \quad \boldsymbol{n}_{, \alpha}=-b_{\alpha \beta} \boldsymbol{a}^{\beta}
$$

respectively, where

$$
\boldsymbol{r}_{; \alpha \beta}=\boldsymbol{r}_{, \alpha \beta}-\Gamma_{\alpha \beta}^{\lambda} \boldsymbol{r}_{, \lambda},
$$

is the (symmetric) second covariant derivative of the surface position field. Here $\Gamma_{\alpha \beta}^{\lambda}$ are the Levi-Civita connection coefficients and $b_{\alpha \beta}$ are the coefficients of the second fundamental form on $\omega$; these are symmetric with respect to interchange of the subscripts, and the latter induce the curvature tensor

$$
\boldsymbol{b}=b_{\alpha \beta} \boldsymbol{a}^{\alpha} \otimes \boldsymbol{a}^{\beta} .
$$

The surfacial gradient of the field $\boldsymbol{n}$ is $\nabla_{s} \boldsymbol{n}=\boldsymbol{n}_{, \alpha} \otimes \boldsymbol{a}^{\alpha}$, in accordance with (11) and $(44)_{2}$. Here the connection coefficients are simply the Christoffel symbols and the connection is therefore metric compatible, i.e., the covariant derivatives of the metric components vanish.

The mean and Gaussian curvatures of $\omega$ are (see (26) 2 and (28) 2 )

$$
H=\frac{1}{2} a^{\alpha \beta} b_{\alpha \beta} \quad \text { and } \quad K=\frac{1}{2} \varepsilon^{\alpha \beta} \varepsilon^{\lambda \mu} b_{\alpha \lambda} b_{\beta \mu},
$$

respectively, where $\varepsilon^{\alpha \beta}=e^{\alpha \beta} / \sqrt{a}$, with $e^{\alpha \beta}=e_{\alpha \beta}$, is the contravariant alternator, and we note the relation

$$
b_{\mu}^{\beta} \tilde{b}^{\mu \alpha}=K a^{\beta \alpha}
$$

where $b_{\mu}^{\beta}=a^{\beta \alpha} b_{\alpha \mu}$ and

$$
\tilde{b}^{\alpha \beta}=\varepsilon^{\alpha \lambda} \varepsilon^{\beta \mu} b_{\lambda \mu}
$$

is the cofactor of the curvature, expressible as

$$
\tilde{b}^{\alpha \beta}=2 H a^{\alpha \beta}-b^{\alpha \beta},
$$

this following on use of the identity

$$
\varepsilon^{\alpha \lambda} \varepsilon^{\beta \mu}=a^{\alpha \beta} a^{\lambda \mu}-a^{\alpha \mu} a^{\beta \lambda} .
$$


The Mainardi-Codazzi equations of surface theory are $b_{\lambda \mu ; \beta}=b_{\lambda \beta ; \mu}$ [Ciarlet 2005], or, more concisely, $\varepsilon^{\beta \mu} b_{\lambda \mu ; \beta}=0$. The metric compatibility of the connection implies that the covariant derivatives of $\varepsilon^{\alpha \lambda}$ vanish and the Mainardi-Codazzi equations are therefore equivalent to

$$
\tilde{b}_{; \beta}^{\alpha \beta}=0 .
$$

5.1. Convected coordinates and surface-fixed coordinates. The literature on lipid bilayers relies exclusively on the use of surface-fixed coordinates in the analysis of the so-called shape equation (see [Ou-Yang et al. 1999] for example). This formalism is entirely analogous to the spatial description of continuum mechanics in which problems are posed on a suitably parametrized fixed region of space. However, as in the latter setting, while this description often affords advantages in the solution of problems, it is a conceptual obstacle to the formulation of theories concerning material bodies. For the latter, convected coordinates that label material points furnish the appropriate alternative.

We encounter precisely the same issue in the mechanics of material surfaces, and thus pause to outline the distinction between parametrizations based on surfacefixed coordinates - analogous to the spatial coordinates of conventional continuum mechanics - and those based on convected coordinates. The relevant developments are due to Scriven [1960] and summarized in Chapter 10 of Aris' book [Aris 1989]. We present the main ideas in the present subsection for the sake of completeness.

Consider configurations of a surface regarded as a material manifold parametrized by a convected coordinate system $\xi^{\alpha}$. This may be identified with the system $\theta^{\alpha}$ of the previous subsection at the value $\epsilon=0$, say, of a time-like parameter $\epsilon$ in a one-parameter family of configurations. The associated surface $\Omega$, with parametric representation $\hat{\boldsymbol{r}}\left(\xi^{\alpha}\right)$, is fixed and may serve as a reference surface in a referential description of the motion. That is, we regard these coordinates as being convected in the sense that they identify, via a map $\boldsymbol{r}=\hat{\boldsymbol{r}}\left(\xi^{\alpha}, \epsilon\right)$, the position, associated with parameter value $\epsilon$, of a material point occupying position $\hat{\boldsymbol{r}}\left(\xi^{\alpha}\right) \in \Omega$ at $\epsilon=0$. This notion may be generalized by regarding $\Omega$ as a surface that is in one-to-one correspondence with that occupied at $\epsilon=0$, so that it need not actually be occupied in the course of the deformation. The connection with the $\theta^{\alpha}$-parametrization of $\omega$ is provided by

$$
\hat{\boldsymbol{r}}\left(\xi^{\alpha}, \epsilon\right)=\boldsymbol{r}\left(\theta^{\alpha}\left(\xi^{\beta}, \epsilon\right), \epsilon\right)
$$

Thus we specify the fixed surface coordinates $\theta^{\alpha}$ as functions of $\xi^{\alpha}$ and $\epsilon$ subject to $\theta^{\alpha}\left(\xi^{\beta}, 0\right)=\xi^{\alpha}$. We assume the relations giving $\theta^{\alpha}$ in terms of $\xi^{\alpha}$ to be invertible, to reflect the notion that at fixed $\epsilon$ the coordinates $\theta^{\alpha}$ can be associated with a unique material point (identified by fixed values of $\xi^{\alpha}$ ). Any function, $f\left(\theta^{\alpha}, \epsilon\right)$, 
say, may then be expressed in terms of convected coordinates as $\hat{f}\left(\xi^{\alpha}, \epsilon\right)$, where

$$
\hat{f}\left(\xi^{\alpha}, \epsilon\right)=f\left(\theta^{\alpha}\left(\xi^{\beta}, \epsilon\right), \epsilon\right) .
$$

The variational derivative of $f$ is its partial derivative with respect to $\epsilon$ in the convected-coordinate representation, i.e., $\dot{f}=\partial \hat{f}\left(\xi^{\alpha}, \epsilon\right) / \partial \epsilon$, whereas its derivative in the fixed-coordinate parametrization is $f_{\epsilon}=\partial f\left(\theta^{\alpha}, \epsilon\right) / \partial \epsilon$; these are related by $\dot{f}=f_{\epsilon}+\left(\theta^{\alpha}\right)^{\bullet} f_{, \alpha}$.

The $\epsilon$-velocity of a material point on $\Omega$ that has been convected by the deformation to $\omega$ is $\boldsymbol{u}=\dot{\boldsymbol{r}}=\partial \hat{\boldsymbol{r}} / \partial \epsilon$. We may write this in terms of components on the natural basis induced by the fixed-coordinate $\theta^{\alpha}$-parametrization:

$$
\boldsymbol{u}=u^{\alpha} \boldsymbol{a}_{\alpha}+w \boldsymbol{n} .
$$

This is related to the derivative $\boldsymbol{r}_{\epsilon}$ by

$$
\boldsymbol{u}=\left(\theta^{\alpha}\right)^{\cdot} \boldsymbol{a}_{\alpha}+\boldsymbol{r}_{\epsilon} .
$$

Following [Aris 1989; Scriven 1960] we adopt the fixed-coordinate parametrization defined by

$$
\frac{d}{d \epsilon} \theta^{\alpha}=u^{\alpha}\left(\theta^{\beta}, \epsilon\right), \quad \theta_{\mid \epsilon=0}^{\alpha}=\xi^{\alpha},
$$

where the derivative is evaluated at fixed $\left\{\xi^{\alpha}\right\}$ and hence equal to $\left(\theta^{\alpha}\right)^{\circ}$. The normal virtual velocity in (55) is then given by

$$
w \boldsymbol{n}=\boldsymbol{r}_{\epsilon},
$$

and the convected and fixed-coordinate derivatives satisfy

$$
\dot{f}=f_{\epsilon}+u^{\alpha} f_{, \alpha} .
$$

We require the Lie derivative of the metric with respect to the velocity. This is simply the variational derivative $\dot{a}_{\alpha \beta}$ expressed in terms of the $\theta^{\alpha}$-parametrization. To this end we adopt convected coordinates $\xi^{\alpha}$ whose values coincide with $\theta^{\alpha}$ at $\epsilon=0$. The two sets of coordinate systems will of course differ at different values of $\epsilon$ due to the fact that material is moving with respect to the $\theta^{\alpha}$-system. Said differently, the material point located at the place with surface coordinates $\theta^{\alpha}$ at $\epsilon=0$ will have different locations at different values of $\epsilon$ and hence be associated with different values of $\theta^{\alpha}$, whereas the values of $\xi^{\alpha}$ remain invariant. Accordingly, while it is always permissible to identify $\xi^{\alpha}$ with $\theta^{\alpha}$ at $\epsilon=0$, say, it is not possible to do so over an interval of $\epsilon$ values. However, for our purposes this limitation is not restrictive. Using $\dot{a}_{\lambda \mu}=\dot{\boldsymbol{a}}_{\lambda} \cdot \boldsymbol{a}_{\mu}+\boldsymbol{a}_{\lambda} \cdot \dot{\boldsymbol{a}}_{\mu}$ and

$$
\dot{\boldsymbol{a}}_{\lambda}=\left(\frac{\partial \boldsymbol{r}}{\partial \theta^{\lambda}}\right)^{\cdot}=\left[\frac{\partial \boldsymbol{r}}{\partial \xi^{\mu}}\left(\frac{\partial \xi^{\mu}}{\partial \theta^{\lambda}}\right)\right]^{\cdot}=\frac{\partial \boldsymbol{u}}{\partial \xi^{\mu}}\left(\frac{\partial \xi^{\mu}}{\partial \theta^{\lambda}}\right)+\frac{\partial \boldsymbol{r}}{\partial \xi^{\mu}}\left(\frac{\partial^{2} \xi^{\mu}}{\partial \theta^{\lambda} \partial \theta^{\alpha}}\right) u^{\alpha},
$$


together with $\partial \xi^{\mu} / \partial \theta^{\lambda}=\delta_{\lambda}^{\mu}$ (the Kronecker delta) and hence $\partial^{2} \xi^{\mu} / \partial \theta^{\lambda} \partial \theta^{\alpha}=0$ at $\epsilon=0$, we derive $\dot{\boldsymbol{a}}_{\alpha}=\partial \boldsymbol{u} / \partial \xi^{\alpha}$ and

$$
\dot{a}_{\lambda \mu}=\boldsymbol{u}_{, \lambda} \cdot \boldsymbol{a}_{\mu}+\boldsymbol{a}_{\lambda} \cdot \boldsymbol{u}_{, \mu},
$$

where $\boldsymbol{u}_{, \lambda}=\partial \boldsymbol{u} / \partial \theta^{\lambda}$ at $\epsilon=0$.

Combining (55) with the Gauss and Weingarten equations yields

$$
\boldsymbol{u}_{, \lambda}=\left(u_{\alpha ; \lambda}-w b_{\alpha \lambda}\right) \boldsymbol{a}^{\alpha}+\left(u^{\alpha} b_{\alpha \lambda}+w_{, \lambda}\right) \boldsymbol{n},
$$

where $\boldsymbol{a}^{\alpha}=a^{\alpha \beta} \boldsymbol{a}_{\beta}$ and $u_{\alpha ; \lambda}$ is the covariant derivative defined by

$$
u_{\alpha ; \lambda}=u_{\alpha, \lambda}-u_{\beta} \Gamma_{\alpha \lambda}^{\beta},
$$

in which $\Gamma_{\alpha \lambda}^{\beta}$ are the connection symbols on $\omega$ pertaining to the induced metric in the $\theta^{\alpha}$-system. Hence the desired expression:

$$
\dot{a}_{\lambda \mu}=u_{\mu ; \lambda}+u_{\lambda ; \mu}-2 w b_{\lambda \mu} .
$$

For example, if $A_{\alpha \beta}$ is the (fixed) metric on the surface $\Omega$ induced by the parametrization $\hat{\boldsymbol{r}}\left(\xi^{\alpha}\right)$, then the areal stretch induced by the deformation is $J=$ $\sqrt{a / A}$, where $A=\operatorname{det}\left(A_{\alpha \beta}\right)$. The fact that the cofactor of $a_{\alpha \beta}$ is $(a) a^{\alpha \beta}$ then implies

$$
\dot{J} / J=\frac{1}{2} a^{\alpha \beta} \dot{a}_{\alpha \beta},
$$

and with (61) this may be reduced to

$$
\dot{J} / J=\boldsymbol{a}^{\alpha} \cdot \boldsymbol{u}_{, \alpha} .
$$

5.2. Congruent configurations of the bilayer and cytoskeleton. This formalism may be adapted to the bilayer/cytoskeleton system by introducing one-parameter families, $\hat{\boldsymbol{r}}_{c}\left(\xi^{\alpha} ; \epsilon_{c}\right)$ and $\hat{\boldsymbol{r}}_{b}\left(\eta^{\alpha} ; \epsilon_{b}\right)$ of cytoskeleton and bilayer deformations respectively, in which $\xi^{\alpha}$ and $\eta^{\alpha}$ are convected coordinates. The surface-fixed coordinates on the cytoskeleton and bilayer are $\theta_{(c)}^{\alpha}\left(\xi^{\alpha} ; \epsilon_{c}\right)$ and $\theta_{(b)}^{\alpha}\left(\eta^{\beta} ; \epsilon_{b}\right)$, respectively. Congruency then implies that (see (53))

$$
\hat{\boldsymbol{r}}_{c}\left(\xi^{\alpha} ; \epsilon_{c}\right)=\boldsymbol{r}\left(\theta_{(c)}^{\alpha}\left(\xi^{\beta} ; \epsilon_{c}\right), \epsilon_{c}\right) \quad \text { and } \quad \hat{\boldsymbol{r}}_{b}\left(\eta^{\alpha} ; \epsilon_{b}\right)=\boldsymbol{r}\left(\theta_{(b)}^{\alpha}\left(\eta^{\beta} ; \epsilon_{b}\right), \epsilon_{b}\right) \text {, }
$$

where $\boldsymbol{r}\left(\theta^{\alpha}, \epsilon\right)$ is the surface-fixed parametrization of $\omega$.

We stipulate that $\xi^{\alpha}=\theta_{(c)}^{\alpha}\left(\xi^{\beta} ; 0\right)$ and $\eta^{\alpha}=\theta_{(b)}^{\alpha}\left(\eta^{\beta} ; 0\right)$; further, that $\theta_{(c)}^{\alpha}\left(\xi^{\beta} ; 0\right)=$ $\theta_{(b)}^{\alpha}\left(\eta^{\beta} ; 0\right)=\theta^{\alpha}$, so that

$$
\hat{\boldsymbol{r}}_{b}\left(\eta^{\alpha} ; 0\right)=\boldsymbol{r}\left(\theta^{\alpha}\right)=\hat{\boldsymbol{r}}_{c}\left(\xi^{\alpha} ; 0\right),
$$

where, for the sake of brevity, we write $\boldsymbol{r}\left(\theta^{\alpha}\right)$ in place of $\boldsymbol{r}\left(\theta^{\alpha}, 0\right)$. In this way we construct convected coordinates $\xi^{\alpha}$ and $\eta^{\alpha}$ that coincide, at $\epsilon_{c}, \epsilon_{b}=0$, with specified surface-fixed coordinates $\theta^{\alpha}$ on $\omega$. This is tantamount to adopting the 
place $\boldsymbol{r}\left(\theta^{\alpha}\right)$ occupied by material points of the bilayer (at $\epsilon_{b}=0$ ) and cytoskeleton (at $\epsilon_{c}=0$ ) as their common reference position.

With reference to $(57)_{1}$ we define the tangential virtual velocities

$$
u^{\alpha}=\frac{d}{d \epsilon_{c}} \theta_{(c) \mid \epsilon_{c}=0}^{\alpha} \quad \text { and } \quad v^{\alpha}=\frac{d}{d \epsilon_{b}} \theta_{(b) \mid \epsilon_{b}=0}^{\alpha},
$$

of the cytoskeleton and bilayer, respectively, and assume, in keeping with congruency, that the normal virtual velocities have a common value, $w$ say:

$$
\left.\frac{\partial \boldsymbol{r}}{\partial \epsilon_{b}}\right|_{\epsilon_{b}=0}=\left.\frac{\partial \boldsymbol{r}}{\partial \epsilon_{c}}\right|_{\epsilon_{c}=0}=w \boldsymbol{n},
$$

(see (58)). Then the virtual velocities of the bilayer and cytoskeleton are

$$
\boldsymbol{u}\left(\theta^{\alpha}\right)=\dot{\boldsymbol{r}}_{b}=u^{\alpha} \boldsymbol{a}_{\alpha}+w \boldsymbol{n},
$$

and

$$
\boldsymbol{v}\left(\theta^{\alpha}\right)=\dot{\boldsymbol{r}}_{c}=v^{\alpha} \boldsymbol{a}_{\alpha}+w \boldsymbol{n},
$$

respectively, where

$$
\dot{\boldsymbol{r}}_{b}=\left.\frac{\partial \hat{\boldsymbol{r}}_{b}}{\partial \epsilon_{b}}\right|_{\epsilon_{b}=0} \quad \text { and } \quad \dot{\boldsymbol{r}}_{c}=\left.\frac{\partial \hat{\boldsymbol{r}}_{c}}{\partial \epsilon_{c}}\right|_{\epsilon_{c}=0} .
$$

The identification of $\boldsymbol{n} \cdot \boldsymbol{u}$ with $\boldsymbol{n} \cdot \boldsymbol{v}$ also features in a model proposed in [Herant and Dembo 2006].

The formula (64) for the variation of the surface metric applies as it stands to the cytoskeleton if the superposed dot is interpreted as a derivative with respect to $\epsilon_{c}$ (evaluated at $\epsilon_{c}=0$ ). It also applies to the bilayer if the superposed dot is interpreted as a derivative with respect to $\epsilon_{b}$ (evaluated at $\epsilon_{b}=0$ ), with $v_{\mu}$ substituted in place of $u_{\mu}$.

To interpret the cytoskeletal deformation tensor $\nabla \boldsymbol{r}_{c}\left(\right.$ see $\left.(7)_{2}\right)$ in this framework, let the patch $\Pi$ be parametrized in the form $\xi\left(\xi^{\alpha}\right)$. This parametrization induces the tangent basis $\boldsymbol{A}_{\alpha}=\boldsymbol{\xi}_{, \alpha}$, metric $A_{\alpha \beta}=\boldsymbol{A}_{\alpha} \cdot \boldsymbol{A}_{\beta}$, dual metric $A^{\alpha \beta}$, and dual basis $\boldsymbol{A}^{\alpha}$. Then,

$$
\nabla \boldsymbol{r}_{c}=\boldsymbol{a}_{\alpha} \otimes \boldsymbol{A}^{\alpha}
$$

and the surfacial Cauchy-Green deformation tensor is

$$
c=a_{\alpha \beta} A^{\alpha} \otimes A^{\beta} .
$$

The areal dilation induced by the deformation is

$$
J_{c}=\sqrt{\operatorname{det} c}=\sqrt{a / A} .
$$




\section{Energy, virtual power and equilibrium}

6.1. Energy and power. To obtain equilibrium equations and edge conditions we invoke the virtual-power principle for the simply-connected patch П. We account for areal incompressibility by extending the energy to unconstrained states and introducing appropriate Lagrange-multiplier fields. Reference may be made to Section 5.10 of [Berdichevsky 2009], for example, for an exposition of this idea together with some of its applications to continuum mechanics. From (34)-(37), the extended energy of the patch is

$$
E=\int_{\Pi}\left[J_{b} W_{b}+J_{c} W_{c}+\lambda_{b}\left(J_{b}-1\right)+\lambda_{c}\left(J_{c}-1\right)\right] d A+\int_{\partial \Pi} \tilde{\mu}\left(J_{b}-1\right) d S,
$$

where $\lambda_{b, c}$ and $\tilde{\mu}$ are Lagrange multiplier fields. We have included a multiplier on the boundary because, as we show below, the tangential and normal derivatives of the virtual bilayer velocity $\boldsymbol{v}$, which figure in the expression for the variation of the energy, are constrained by areal incompressibility. To our knowledge this effect has not been discussed in the literature on bilayers. However, similar terms are known to play a role in the mechanics of continua of second grade [Guven et al. 2019; Steigmann 2018; Wang and Pipkin 1986] — as exemplified by lipid bilayers - in the presence of constraints on the first-order gradients.

Having proposed an expression for the extended energy, we identify equilibria with those states that satisfy

$$
\dot{E}=P \text {, }
$$

where $P$ is the virtual power imparted to the patch. The form that this power takes is deduced in the course of the ensuing development. Here the superposed dot refers to a Gateaux derivative with respect to either $\epsilon_{c}$ or $\epsilon_{b}$ (evaluated at $\epsilon_{c}$ and $\epsilon_{b}$ equal to zero) or to both simultaneously.

6.2. Tangential equilibrium of the cytoskeletal membrane. For example, consider variations that preserve the bilayer configuration. These are $\boldsymbol{u}\left(\theta^{\alpha}\right)=u^{\alpha} \boldsymbol{a}_{\alpha}$ and $\boldsymbol{v}=\mathbf{0}$, and yield

$$
\dot{E}=\int_{\pi_{c}}\left[\dot{W}_{c}+\left(W_{c}+\lambda_{c}\right) \dot{J}_{c} / J_{c}\right] d a,
$$

in which variation of $\lambda_{c}$ has been suppressed as this merely returns the areal incompressibility constraint. In the extended (unconstrained) formalism, $J_{c} W_{c}$ is the cytoskeletal energy density on $\Pi$. Thus, in the case of isotropy, for example, we make the identification

$$
J_{c} W_{c}=\varpi(I), \quad \text { with } \quad I=a_{\lambda \mu} A^{\lambda \mu},
$$


which reduces to (42) when the constraint is in effect. This depends via (75) and (76) on the surfacial Cauchy-Green tensor $\boldsymbol{c}$ and thus evolves in response to variations $\dot{a}_{\alpha \beta}$ of the surface metric. Accordingly, we write

$$
\left(J_{c} W_{c}\right)^{\cdot}=\frac{1}{2} J_{c} \Sigma^{\alpha \beta} \dot{a}_{\alpha \beta}, \quad \text { with } \quad \frac{1}{2} J_{c} \Sigma^{\alpha \beta}=\left(J_{c} W_{c}\right)_{c} \cdot A^{\alpha} \otimes A^{\beta},
$$

which we combine with (65) to obtain

$$
\dot{W}_{c}+\left(W_{c}+\lambda_{c}\right) \dot{J}_{c} / J_{c}=\frac{1}{2} \sigma^{\alpha \beta} \dot{a}_{\alpha \beta}, \quad \text { with } \quad \sigma^{\alpha \beta}=\Sigma^{\alpha \beta}+\lambda_{c} a^{\alpha \beta} .
$$

We note that $\Sigma^{\alpha \beta}=\Sigma^{\beta \alpha}$, and thus $\sigma^{\alpha \beta}=\sigma^{\beta \alpha}$, by virtue of the symmetry of $\left(J_{c} W_{c}\right)_{c}$. For example, in the case of isotropy, we have from (75) and (80) that $\left(J_{c} W_{c}\right)_{c}=\varpi^{\prime}(I) \mathbf{1}_{\Pi}$, yielding

$$
J_{c} \Sigma^{\alpha \beta}=2 \varpi^{\prime}(I) A^{\alpha \beta} .
$$

Combining this symmetry with (64) (with $w=0$ ) we derive $\frac{1}{2} \sigma^{\alpha \beta} \dot{a}_{\alpha \beta}=\sigma^{\alpha \beta} u_{\alpha ; \beta}$ and then convert (79) via Stokes' theorem to

$$
\dot{E}=\int_{\partial \pi_{c}} \sigma^{\alpha \beta} v_{\beta} u_{\alpha} d s-\int_{\pi_{c}} \sigma_{; \beta}^{\alpha \beta} u_{\alpha} d a,
$$

where $v_{\beta}=\varepsilon_{\beta \alpha} \tau^{\alpha}$, in which $\tau^{\alpha}=d \theta^{\alpha} / d s$ are the components of the rightward unit normal to $\partial \pi_{c}$ with arclength parametrization $\theta^{\alpha}(s)$; i.e., $\boldsymbol{v}=\boldsymbol{\tau} \times \boldsymbol{n}$, where $\boldsymbol{\tau}=d \boldsymbol{r}\left(\theta^{\alpha}(s)\right) / d s$ and $\boldsymbol{n}$ respectively are the unit tangent to $\partial \pi_{c}$ and the unit surface normal.

From (78) it follows that the virtual power is of the form

$$
P=\int_{\partial \pi_{c}} t_{(c)}^{\alpha} u_{\alpha} d s+\int_{\pi_{c}} g_{(c)}^{\alpha} u_{\alpha} d a
$$

and, with no further restrictions on $u_{\alpha}$, that

$$
\sigma_{; \beta}^{\alpha \beta}+g_{(c)}^{\alpha}=0, \quad \text { in } \quad \pi_{c} \text { and } t_{(c)}^{\alpha}=\sigma^{\alpha \beta} v_{\beta}, \quad \text { on } \partial \pi_{c},
$$

in which $g_{(c)}^{\alpha}$ and $t_{(c)}^{\alpha}$ respectively are the distributed tangential force (per unit area) and the tangential edge traction (force per unit length) acting on the cytoskeleton. From these relations it is clear that $\sigma^{\alpha \beta}$ plays the role of the cytoskeletal Cauchy stress. Equation $(82)_{2}$ then yields the interpretation of $\lambda_{c}$ as a reactive surface tension. Here, to compensate for having suppressed variation with respect to the multiplier $\lambda_{c}$, it is necessary to impose $J_{c}=1$ a posteriori. Thus, in the case of isotropy, we use (82) $)_{2}$ in (86) with

$$
\Sigma^{\alpha \beta}=2 \varpi^{\prime}(I) A^{\alpha \beta} .
$$


6.3. Variational derivative of the bilayer energy. We pause to discuss some formulae of a general nature valid for arbitrary bilayer virtual velocities $\boldsymbol{v}$ and subsequently specialize these to derive the tangential equilibrium equations.

First we note that because $J_{b}$ and $W_{b}$ depend on the surface position field through its first and second derivatives with respect to the coordinates, it follows that there are vector fields $\boldsymbol{N}^{\alpha}$ and $\boldsymbol{M}^{\alpha \beta}$ such that

$$
\dot{W}_{b}+\left(W_{b}+\lambda_{b}\right) \dot{J}_{b} / J_{b}=N^{\alpha} \cdot \boldsymbol{v}_{, \alpha}+M^{\alpha \beta} \cdot \boldsymbol{v}_{; \alpha \beta},
$$

where $\boldsymbol{v}=\dot{\boldsymbol{r}}_{b}$ is the virtual velocity and $\boldsymbol{v}_{; \alpha \beta}=\boldsymbol{v}_{, \alpha \beta}-\Gamma_{\alpha \beta}^{\lambda} \boldsymbol{v}_{, \lambda}$ is the second covariant derivative of $\boldsymbol{v}$. This is symmetric in the subscripts; therefore, no generality is lost by imposing $\boldsymbol{M}^{\alpha \beta}=\boldsymbol{M}^{\beta \alpha}$.

For example [Agrawal and Steigmann 2009],

$$
\dot{H}=\frac{1}{2} a^{\alpha \beta} \boldsymbol{n} \cdot \boldsymbol{v}_{; \alpha \beta}-b^{\alpha \beta} \boldsymbol{a}_{\beta} \cdot \boldsymbol{v}_{, \alpha} \quad \text { and } \quad \dot{K}=\tilde{b}^{\alpha \beta} \boldsymbol{n} \cdot \boldsymbol{v}_{; \alpha \beta}-2 K \boldsymbol{a}^{\alpha} \cdot \boldsymbol{v}_{, \alpha},
$$

whereas (see (66))

$$
\dot{J}_{b} / J_{b}=\boldsymbol{a}^{\alpha} \cdot \boldsymbol{v}_{, \alpha} .
$$

Using $\dot{W}_{b}=2 \kappa H \dot{H}+\bar{\kappa} \dot{K}$ (from (36) $)$ we thus derive

$$
\boldsymbol{N}^{\mu}=N^{\mu \beta} \boldsymbol{a}_{\beta} \quad \text { and } \quad \boldsymbol{M}^{\mu \beta}=M^{\mu \beta} \boldsymbol{n},
$$

with

$$
N^{\mu \beta}=\left(\lambda_{b}+\kappa H^{2}-\bar{\kappa} K\right) a^{\mu \beta}-2 \kappa H b^{\mu \beta} \quad \text { and } \quad M^{\mu \beta}=\kappa H a^{\mu \beta}+\bar{\kappa} \tilde{b}^{\mu \beta} .
$$

Proceeding, we have

$$
\boldsymbol{N}^{\alpha} \cdot \boldsymbol{v}_{, \alpha}+\boldsymbol{M}^{\alpha \beta} \cdot \boldsymbol{v}_{; \alpha \beta}=\varphi_{; \alpha}^{\alpha}-\boldsymbol{v} \cdot \boldsymbol{T}_{; \alpha}^{\alpha},
$$

where

$$
\boldsymbol{T}^{\alpha}=\boldsymbol{N}^{\alpha}-\boldsymbol{M}_{; \beta}^{\alpha \beta},
$$

with

$$
\boldsymbol{M}_{; \beta}^{\beta \alpha}=M_{; \beta}^{\beta \alpha} \boldsymbol{n}-M^{\beta \alpha} b_{\beta}^{\mu} \boldsymbol{a}_{\mu},
$$

and

$$
\varphi^{\alpha}=\boldsymbol{T}^{\alpha} \cdot \boldsymbol{v}+\boldsymbol{M}^{\alpha \beta} \cdot \boldsymbol{v}_{, \beta},
$$

in which (91), (94) and (95) together give

$$
\boldsymbol{T}^{\alpha}=\left(N^{\alpha \mu}+M^{\alpha \beta} b_{\beta}^{\mu}\right) \boldsymbol{a}_{\mu}-M_{; \beta}^{\alpha \beta} \boldsymbol{n} .
$$

Combining (88) and (96) with Stokes' theorem furnishes

$$
\int_{\pi_{b}}\left[\dot{W}_{b}+\left(W_{b}+\lambda_{b}\right) \dot{J}_{b} / J_{b}\right] d a=\int_{\partial \pi_{b}} \varphi^{\alpha} v_{\alpha} d s-\int_{\pi_{b}} \boldsymbol{v} \cdot \boldsymbol{T}_{; \alpha}^{\alpha} d a,
$$


where $\boldsymbol{v}=v_{\alpha} \boldsymbol{a}^{\alpha}$ is the exterior unit normal $\partial \pi_{b}$ and

$$
\boldsymbol{v} \cdot \boldsymbol{T}_{; \alpha}^{\alpha}=v_{\mu} \boldsymbol{a}^{\mu} \cdot \boldsymbol{T}_{; \alpha}^{\alpha}+w \boldsymbol{n} \cdot \boldsymbol{T}_{; \alpha}^{\alpha},
$$

with

$$
\boldsymbol{a}^{\mu} \cdot \boldsymbol{T}_{; \alpha}^{\alpha}=\left(N^{\alpha \mu}+M^{\alpha \beta} b_{\beta}^{\mu}\right)_{; \alpha}+M_{; \beta}^{\alpha \beta} b_{\alpha}^{\mu},
$$

and

$$
\boldsymbol{n} \cdot \boldsymbol{T}_{; \alpha}^{\alpha}=\left(N^{\alpha \mu}+M^{\alpha \beta} b_{\beta}^{\mu}\right) b_{\mu \alpha}-M_{; \beta \alpha}^{\beta \alpha} .
$$

In the first term on the right-hand side of (98) we use the normal-tangential decomposition

$$
\boldsymbol{v}_{, \beta}=\tau_{\beta} \boldsymbol{v}^{\prime}+v_{\beta} \boldsymbol{v}_{v},
$$

where $\boldsymbol{\tau}=\tau_{\alpha} \boldsymbol{a}^{\alpha}=\boldsymbol{n} \times \boldsymbol{v}$ is the unit tangent to $\partial \pi_{b}, \boldsymbol{v}^{\prime}=\tau^{\alpha} \boldsymbol{v}_{, \alpha}=d \boldsymbol{v} / d s$ is the tangential derivative of $\boldsymbol{v}$, and $\boldsymbol{v}_{v}=v^{\alpha} \boldsymbol{v}_{, \alpha}$ is the normal derivative. The term involving the tangential derivative is integrated by parts. If $\partial \pi_{b}$ is piecewise smooth in the sense that its tangent $\tau$ is piecewise continuous, with discontinuities at a finite number of corners, then

$$
\begin{aligned}
\int_{\partial \pi_{b}} \varphi^{\alpha} v_{\alpha} d s= & \int_{\partial \pi_{b}}\left(\left\{\boldsymbol{T}^{\alpha} v_{\alpha}-\left(\boldsymbol{M}^{\alpha \beta} v_{\alpha} \tau_{\beta}\right)^{\prime}\right\} \cdot \boldsymbol{v}+\boldsymbol{M}^{\alpha \beta} v_{\alpha} v_{\beta} \cdot \boldsymbol{v}_{v}\right) d s \\
& -\sum \boldsymbol{M}^{\alpha \beta}\left[v_{\alpha} \tau_{\beta}\right]_{i} \cdot \boldsymbol{v}_{i},
\end{aligned}
$$

in which the square bracket refers to the forward jump as a corner of the boundary is traversed, and the sum ranges over all corners. Thus, $[\cdot]=(\cdot)_{+}-(\cdot)_{-}$, where the subscripts ${ }_{ \pm}$respectively identify limits as a corner located at arclength station $s$ is approached through larger and smaller values of arclength.

6.3.1. Tangential bilayer equilibrium. Consider variations with $\boldsymbol{v}$ and $\boldsymbol{v}_{v}$ vanishing on $\partial \pi_{b}$ (and at corners) that preserve the configuration of the cytoskeleton, i.e., $\boldsymbol{u}=\mathbf{0}$ and $\boldsymbol{v}=v_{\mu} \boldsymbol{a}^{\mu}$ in the interior of $\pi_{b}$. For these we have

$$
\dot{E}=\int_{\pi_{b}}\left[\dot{W}_{b}+\left(W_{b}+\lambda_{b}\right) \dot{J}_{b} / J_{b}\right] d a=-\int_{\pi_{b}} v_{\mu} \boldsymbol{a}^{\mu} \cdot \boldsymbol{T}_{; \alpha}^{\alpha} d a,
$$

in which variation of $\lambda_{b}$ has been suppressed, and it follows, from (78), that the virtual power is of the form

$$
P=\int_{\pi_{b}} g_{(b)}^{\mu} v_{\mu} d a
$$

where $g_{(b)}^{\mu}$ is a tangential force (per unit area) acting on the bilayer. Because $v_{\mu}$ is unrestricted, we arrive at

$$
\left(N^{\alpha \mu}+M^{\alpha \beta} b_{\beta}^{\mu}\right)_{; \alpha}+M_{; \beta}^{\alpha \beta} b_{\alpha}^{\mu}+g_{(b)}^{\mu}=0, \quad \text { in } \quad \pi_{b} .
$$


To reduce this we use (50), (52) and (92) to infer that

$$
N^{\alpha \mu}+M^{\alpha \beta} b_{\beta}^{\mu}=\left(\lambda_{b}+\kappa H^{2}\right) a^{\alpha \mu}-\kappa H b^{\alpha \mu},
$$

with divergence

$$
\left(N^{\alpha \mu}+M^{\alpha \beta} b_{\beta}^{\mu}\right)_{; \alpha}=a^{\alpha \mu}\left(\lambda_{b}\right)_{, \alpha}+2 \kappa H a^{\alpha \mu} H_{, \alpha}-\kappa b^{\alpha \mu} H_{, \alpha}-\kappa H b_{; \alpha}^{\alpha \mu},
$$

and combination with (see $\left.(92)_{2}\right)$

$$
M_{; \beta}^{\alpha \beta}=\kappa a^{\alpha \beta} H_{, \beta},
$$

furnishes

$$
\left(N^{\alpha \mu}+M^{\alpha \beta} b_{\beta}^{\mu}\right)_{; \alpha}+M_{; \beta}^{\alpha \beta} b_{\alpha}^{\mu}=a^{\alpha \mu}\left(\lambda_{b}\right)_{, \alpha}+\kappa H\left(2 a^{\alpha \mu} H_{, \alpha}-b_{; \alpha}^{\alpha \mu}\right),
$$

in which the second parenthetical term on the right is $\left(2 H a^{\mu \alpha}-b^{\mu \alpha}\right)_{; \alpha}=\tilde{b}_{; \alpha}^{\mu \alpha}$. Then, with (52), equation (110) reduces simply to

$$
a^{\alpha \mu}\left(\lambda_{b}\right)_{, \alpha}+g_{(b)}^{\mu}=0 .
$$

6.3.2. Comoving bilayer and cytoskeleton. If the cytoskeleton is anchored to the bilayer such as to convect with it, then $\boldsymbol{u}=\boldsymbol{v}$ in $\pi^{*}=\pi_{b} \cap \pi_{c}$. Choosing variations such that $\boldsymbol{u}, \boldsymbol{v}$ and $\boldsymbol{v}_{v}$ vanish on $\partial \pi^{*}$ and $\boldsymbol{v}=v_{\mu} \boldsymbol{a}^{\mu}$ in $\pi^{*}$, with $\boldsymbol{u}, \boldsymbol{v}$ vanishing in $\omega \backslash \pi^{*}$, we obtain

$$
\dot{E}=\int_{\pi^{*}}[\dot{W}+(W+\lambda) \dot{J} / J] d a,
$$

with $W=W_{b}+W_{c}, \lambda=\lambda_{b}+\lambda_{c}$ and $\dot{J} / J=v_{; \mu}^{\mu}$. We could proceed from this statement to derive the relevant balance equation directly, but it is more illuminating to combine (84) and (104) to arrive at

$$
\dot{E}=-\int_{\pi^{*}}\left\{\sigma_{; \alpha}^{\mu \alpha}+a^{\mu \alpha}\left(\lambda_{b}\right)_{, \alpha}\right\} v_{\mu} d a .
$$

The associated virtual power therefore has the form

$$
P=\int_{\pi^{*}} g^{\mu} v_{\mu} d a,
$$

and with $v_{\mu}$ unrestricted, (86) $)_{1}$ and (111) then deliver

$$
g^{\mu}=g_{(b)}^{\mu}+g_{(c)}^{\mu} .
$$

Equivalently,

$$
\left(\Sigma^{\mu \alpha}+\lambda a^{\mu \alpha}\right)_{; \alpha}+g^{\mu}=0,
$$

in which the term in parentheses is the effective Cauchy stress for the bilayer/cytoskeleton composite subjected to a net tangential force $g^{\mu}$. 
6.4. Normal equilibrium of the bilayer and cytoskeleton. Having exhausted the consequences of the virtual-power statement for tangential variations, we proceed next to normal variations. In view of (71) and (72), these involve the bilayer and cytoskeleton together. Taking variations as in the previous subsection, now with $\boldsymbol{u}=\boldsymbol{v}=w \boldsymbol{n}$, with reference to (64), (82) 1 and (98) we obtain

$$
\dot{E}=-\int_{\pi^{*}} w\left(\sigma^{\alpha \beta} b_{\alpha \beta}+\boldsymbol{n} \cdot \boldsymbol{T}_{; \alpha}^{\alpha}\right) d a,
$$

and conclude that the associated power has the form

$$
P=\int_{\pi^{*}} p w d a,
$$

where $p$ is the net lateral pressure acting on the surface in the direction of $\boldsymbol{n}$. Thus, with (101) and with $w$ unrestricted, we arrive at

$$
\sigma^{\alpha \beta} b_{\alpha \beta}+\left(N^{\alpha \mu}+M^{\alpha \beta} b_{\beta}^{\mu}\right) b_{\mu \alpha}-M_{; \beta \alpha}^{\beta \alpha}+p=0, \quad \text { in } \pi^{*} .
$$

To reduce this we use (92), finding that

$$
\left(N^{\alpha \mu}+M^{\alpha \beta} b_{\beta}^{\mu}\right) b_{\mu \alpha}=2 \lambda_{b} H+2 \kappa H^{3}-\kappa H b^{\alpha \mu} b_{\mu \alpha} .
$$

The final term on the right is $b^{\alpha \mu} b_{\mu \alpha}=\operatorname{tr}\left(\boldsymbol{b}^{2}\right)$, and with (29) and (109), equation (119) becomes

$$
\kappa\left[\Delta H+2 H\left(H^{2}-K\right)\right]-2 \lambda_{b} H=\sigma^{\alpha \beta} b_{\alpha \beta}+p,
$$

where

$$
\Delta H=a^{\alpha \beta} H_{; \alpha \beta}=\frac{1}{\sqrt{a}}\left(\sqrt{a} a^{\alpha \beta} H_{, \beta}\right)_{, \alpha},
$$

is the surfacial Laplacian of $H$.

Equation (121) is the classical shape equation for lipid bilayers in which the right-hand side is the pressure transmitted to the bilayer [Dharmavaram and Healey 2015; Jenkins 1977; Nitsche 1993]. Thus the cytoskeleton, if curved, transmits an effective pressure to the bilayer that persists when the net pressure $p$ acting on the system vanishes. Vice versa, the bilayer transmits an equal but opposite pressure to the cytoskeleton.

We may rewrite (121) in the form

$$
\kappa\left[\Delta H+2 H\left(H^{2}-K\right)\right]=\left(\Sigma^{\alpha \beta}+\lambda a^{\alpha \beta}\right) b_{\alpha \beta}+p .
$$

This is the appropriate equation to use if the cytoskeleton convects with the bilayer because the parenthetical term on the right is then subject to (116), and in this setting extends the system obtained in [Guven et al. 2019] for strain-free deformations in which the entire metric, and not just the local areal stretch, is constrained, 
with $T^{\alpha \beta}=\Sigma^{\alpha \beta}+\lambda a^{\alpha \beta}$, in which the $\Sigma^{\alpha \beta}$ are constitutively indeterminate, then serving as the operative Lagrange multipliers.

6.5. Edge conditions. Boundary conditions are of limited relevance in this subject because bilayers typically form closed surfaces. Nevertheless, in the present approach based on the notion of patchwise equilibrium, they deliver expressions for the various actions at the edge of a patch which are of independent interest. Further, a number of models that entail boundary interactions are available in the literature [Agrawal and Steigmann 2009; Guven et al. 2019; Rosso and Virga 1999].

With the foregoing Euler equations satisfied on $\omega$, the variation of the energy reduces, with the aid of (77), (84) and (98), to

$$
\dot{E}=\int_{\partial \pi^{*}}\left(\sigma^{\alpha \beta} v_{\beta} u_{\alpha}+\varphi^{\alpha} v_{\alpha}+\mu \dot{J}_{b} / J_{b}\right) d s,
$$

where $\mu d s=\left(\tilde{\mu} J_{b}\right) d S$ and $\varphi^{\alpha}$ is defined by (96).

We note, from (90) and (102), that the constraint $J_{b}=1$ yields $\boldsymbol{\tau} \cdot \boldsymbol{v}^{\prime}+\boldsymbol{v}$. $\boldsymbol{v}_{v}=0$, implying that the normal and tangential derivatives of $\boldsymbol{v}$ on $\partial \pi^{*}$ are not independent. Because $\boldsymbol{v}^{\prime}$ is determined by $\boldsymbol{v}_{\mid \partial \pi^{*}}$, it follows that $\boldsymbol{v}$ and $\boldsymbol{v}_{v}$ cannot be specified independently. In the extended formulation, this restriction is relaxed and an associated Lagrange multiplier $\mu$ is introduced. Then, with (103) we obtain

$$
\begin{aligned}
\dot{E}=\int_{\partial \pi^{*}}\left\{\sigma^{\alpha \beta} v_{\beta} u_{\alpha}+\left[\boldsymbol{T}^{\alpha} v_{\alpha}-\left(\boldsymbol{M}^{\alpha \beta} v_{\alpha} \tau_{\beta}+\mu \boldsymbol{\tau}\right)^{\prime}\right] \cdot \boldsymbol{v}+\left(\boldsymbol{M}^{\alpha \beta} v_{\alpha} v_{\beta}+\mu \boldsymbol{v}\right) \cdot \boldsymbol{v}_{\nu}\right\} d s \\
-\sum\left[\boldsymbol{M}^{\alpha \beta} v_{\alpha} \tau_{\beta}+\mu \boldsymbol{\tau}\right]_{i} \cdot \boldsymbol{v}_{i} .
\end{aligned}
$$

The virtual power is thus expressible in the form

$$
P=\int_{\partial \pi^{*}}\left(\boldsymbol{t}_{c} \cdot \boldsymbol{u}+\boldsymbol{t}_{b} \cdot \boldsymbol{v}+\boldsymbol{\mu} \cdot \boldsymbol{v}_{v}\right) d s+\sum \boldsymbol{f}_{i} \cdot \boldsymbol{v}_{i},
$$

where $\boldsymbol{t}_{c}, \boldsymbol{t}_{b}, \boldsymbol{\mu}$ and $\boldsymbol{f}_{i}$ respectively are the cytoskeletal and bilayer tractions and the double force and corner forces acting on the bilayer patch. Accordingly,

$$
\begin{gathered}
\boldsymbol{t}_{c}=\sigma^{\alpha \beta} v_{\beta} \boldsymbol{a}_{\beta}, \quad \boldsymbol{t}_{b}=\boldsymbol{T}^{\alpha} v_{\alpha}-\left(M^{\alpha \beta} v_{\alpha} \tau_{\beta} \boldsymbol{n}+\mu \boldsymbol{\tau}\right)^{\prime}, \\
\boldsymbol{\mu}=M \boldsymbol{n}+\mu \boldsymbol{v} \text { and } \boldsymbol{f}_{i}=-\left[M^{\alpha \beta} v_{\alpha} \tau_{\beta} \boldsymbol{n}+\mu \boldsymbol{\tau}\right]_{i}, \text { with } M=M^{\alpha \beta} v_{\alpha} v_{\beta} .
\end{gathered}
$$

The first of these is just the condition $(86)_{2}$ on $\partial \pi^{*}$.

The couple acting on the interior of $\partial \pi^{*}$ is

$$
c=r \times t+r_{v} \times \mu,
$$

where $\boldsymbol{t}=\boldsymbol{t}_{b}+\boldsymbol{t}_{c}$ is the net traction and $\boldsymbol{r}_{v}=v^{\alpha} \boldsymbol{r}_{, \alpha}=\boldsymbol{v}$. Thus,

$$
c-r \times t=-M \tau,
$$


a pure bending couple acting at the edge that does not involve the multiplier $\mu$. However, it is not appropriate to assign the couple in a boundary-value problem. Rather, information about $\mu$ is furnished by the specification of the double force [Toupin 1962].

If the bilayer and cytoskeleton are comoving, then (127) 3,4 remain in effect but $(127)_{1,2}$ are replaced by the single equation

$$
\boldsymbol{t}=\left(\boldsymbol{T}^{\alpha}+\sigma^{\alpha \beta} \boldsymbol{a}_{\beta}\right) v_{\alpha}-\left(M^{\alpha \beta} v_{\alpha} \tau_{\beta} \boldsymbol{n}+\mu \boldsymbol{\tau}\right)^{\prime} .
$$

\section{Legendre-Hadamard conditions}

If the cytoskeleton convects with the bilayer, then because the effective energy involves the spatial derivatives of a single deformation field through the second order, the operative Legendre-Hadamard necessary condition for energy minimizers entails perturbation of the latter only, at fixed values of the first derivatives [Hilgers and Pipkin 1993]. Because the cytoskeletal energy involves only first derivatives, the operative Legendre-Hadamard condition then involves the bilayer energy alone. For the energy (36) $)_{1}$, this yields the nonnegativity of the bending modulus $k$ [Agrawal and Steigmann 2008], as implied by $(24)_{1}$ and (31) ${ }_{1}$.

If the cytoskeleton and bilayer are not comoving, then the membrane-theoretic version of the Legendre-Hadamard condition is applicable, and implies that, at an arbitrary material point $p$, say, the cytoskeletal energy, regarded as a function of $\nabla \boldsymbol{r}_{c}$, is locally convex with respect to perturbations of the form

$$
\boldsymbol{u}_{, \alpha}=\boldsymbol{a} k_{\alpha},
$$

i.e.,

$$
a^{\mu} k_{\alpha}=u_{; \alpha}^{\mu}-w b_{\alpha}^{\mu} \quad \text { and } \quad a k_{\alpha}=u^{\mu} b_{\mu \alpha}+w_{, \alpha},
$$

with $a^{\mu}=\boldsymbol{a} \cdot \boldsymbol{a}^{\mu}$ and $a=\boldsymbol{a} \cdot \boldsymbol{n}$, subject tof $\boldsymbol{a}^{\alpha} \cdot \boldsymbol{a} k_{\alpha}=0$ on account of areal incompressibility (see (66)). Thus, areal incompressibility imposes the restriction

$$
a^{\alpha \beta} a_{\beta} k_{\alpha}=0,
$$

where $a_{\beta}=a_{\beta \mu} a^{\mu}$.

The operative Legendre-Hadamard condition is [Steigmann 1990]

$$
\boldsymbol{a} \cdot\left(\boldsymbol{E}^{\alpha \beta} k_{\alpha} k_{\beta}\right) \boldsymbol{a} \geq 0,
$$

for arbitrary $\boldsymbol{a} k_{\alpha}$ subject to (133), where

$$
\boldsymbol{E}^{\alpha \beta}=2 \frac{\partial W}{\partial a_{\alpha \beta}} \boldsymbol{I}+4 \frac{\partial^{2} W}{\partial a_{\alpha \mu} \partial a_{\beta \lambda}} \boldsymbol{a}_{\mu} \otimes \boldsymbol{a}_{\lambda},
$$


in which $W\left(a_{\alpha \beta}\right)=W_{c}\left(a_{\mu \lambda} \boldsymbol{A}^{\mu} \otimes \boldsymbol{A}^{\lambda}\right)$. Then, with (81) 1 , specialized to $J_{c}=1$, we require

$$
\Sigma^{\alpha \beta} k_{\alpha} k_{\beta}|\boldsymbol{a}|^{2}+4 \frac{\partial^{2} W}{\partial a_{\alpha \mu} \partial a_{\beta \lambda}} a_{\mu} k_{\alpha} a_{\lambda} k_{\beta} \geq 0
$$

where $\Sigma^{\alpha \beta}$ is the constitutively determined part of the cytoskeletal Cauchy stress.

This condition yields a nontrivial restriction on $W$ even if the bilayer remains undisturbed; i.e., if $w=0$.

The choice $\boldsymbol{a}=a \boldsymbol{n}\left(a_{\beta}=0\right)$ conforms to (133) and reduces (136) to

$$
\Sigma^{\alpha \beta} k_{\alpha} k_{\beta} \geq 0,
$$

implying that the energetic part of the stress is positive semidefinite in energy minimizing states. In the absence of constraints, this implies, in accordance with a restriction proposed in [Stamenovic 2006], that the Cauchy stress is positive semidefinite.

For example, in the case of isotropy (see (87)), (137) reduces to $\varpi^{\prime}(I)|\boldsymbol{k}|^{2} \geq 0$, where $|\boldsymbol{k}|^{2}=A^{\alpha \beta} k_{\alpha} k_{\beta}$, and is thus satisfied if and only if

$$
\varpi^{\prime}(I) \geq 0,
$$

whereas the full Legendre-Hadamard inequality (136), in the case of isotropy, is

$$
\varpi^{\prime}(I)|\boldsymbol{a}|^{2}|\boldsymbol{k}|^{2}+2 \varpi^{\prime \prime}(I)\left(k^{\alpha} a_{\alpha}\right)^{2} \geq 0,
$$

with $k^{\alpha}=A^{\alpha \beta} k_{\beta}$.

\section{Equivalent monolayers with spontaneous curvature}

8.1. Equilibrium of monolayers. We expect the conforming cytoskeleton to confer asymmetry in the bending response of the bilayer/cytoskeleton composite, whereas that of an isolated bilayer is symmetric in the sense that the energy $(36)_{1}$ is the invariant under $\boldsymbol{b} \rightarrow-\boldsymbol{b}$. Asymmetric bending is also a feature of conventional monolayers, consisting of one sheet of oriented lipids instead of two of opposing orientation (Figure 1). Conventionally, this asymmetry is modelled by introducing a spontaneous curvature $C\left(\theta^{\alpha}\right)$ [Ou-Yang et al. 1999] via the energy

$$
W\left(H, K ; \theta^{\alpha}\right)=\kappa(H-C)^{2}+\bar{\kappa} K .
$$

The existence of these distinct models of asymmetric bending leads us to search for conditions under which they might be equivalent.

Proceeding as in Section 6.3, we derive (97) but with (92) replaced by

$$
\begin{gathered}
N^{\alpha \mu}=\left\{\lambda_{m}+\kappa(H-C)^{2}-\bar{\kappa} K\right\} a^{\alpha \mu}-2 \kappa(H-C) b^{\alpha \mu}, \\
\text { and } M^{\alpha \mu}=\kappa(H-C) a^{\alpha \mu}+\bar{\kappa} \tilde{b}^{\alpha \mu},
\end{gathered}
$$


where $\lambda_{m}$ is a Lagrange multiplier associated with the areal incompressibility of the monolayer. Then with some labor we find that (111) is replaced by

$$
a^{\alpha \mu}\left[\left(\lambda_{m}\right)_{, \alpha}-2 \kappa(H-C) C_{, \alpha}\right]+g_{(m)}^{\mu}=0,
$$

where $g_{(m)}^{\mu}$ is a tangential distribution of force on the monolayer; and, in the absence of the cytoskeleton, that (121) is replaced by

$$
\kappa\left[\Delta(H-C)+2(H-C)\left(2 H^{2}-K\right)-2 H(H-C)^{2}\right]-2 \lambda_{m} H=p,
$$

where $p$ is the pressure exerted on the monolayer.

Evidently, (142) corresponds to (111) if $C_{, \alpha}$ vanishes, i.e., if the spontaneous curvature is uniform. In this case we have

$$
\kappa\left[\Delta H+2 H\left(H^{2}-K\right)\right]-2 \lambda_{m} H=p+2 \kappa C(C H-K),
$$

which corresponds to (121), provided that $\lambda_{m}=\lambda_{b}$ and the cytoskeletal stress $\sigma^{\alpha \beta}$ satisfies

$$
\sigma^{\alpha \beta} b_{\alpha \beta}=2 \kappa C(C H-K) .
$$

Equations (47) and (49) furnish $2 H=a^{\alpha \beta} b_{\alpha \beta}$ and $2 K=\tilde{b}^{\alpha \beta} b_{\alpha \beta}$, and so a sufficient condition for such correspondence is

$$
\sigma^{\alpha \beta}=\kappa C\left(C a^{\alpha \beta}-\tilde{b}^{\alpha \beta}\right),
$$

provided that no tangential force is acting on the cytoskeleton. For, this expression for the stress is automatically divergence-free and (86) ${ }_{1}$ requires that the tangential force vanish.

We observe, noting (123), that this same correspondence may be established between the monolayer and the comoving cytoskeleton if $\lambda_{m}=0$ and if $\lambda_{c}=\lambda$ in $(82)_{2}$.

These correspondences must be qualified by the fact that the constitutive response of the cytoskeleton cannot be expected to yield (146) in general. Nevertheless, in the absence of tangential forces, the latter allows us to dispense with $(86)_{1}$ or (116), which would otherwise pose significant obstacles to analysis. Thus, we view (146) simply as a device for generating potential solutions by selecting from among a number of explicit solutions that are available for monolayers with constant spontaneous curvature [Ou-Yang et al. 1999]. Remarkably, these include the characteristic biconcave discoid shape of red-blood cells in equilibrium.

8.2. Biconcave discoid. Consider a surface of revolution described by

$$
\boldsymbol{r}\left(\theta^{\alpha}\right)=r \boldsymbol{e}_{r}(\theta)+z(r) \boldsymbol{k},
$$

where $r\left(=\theta^{1}\right)$ is the radius from the symmetry axis directed along the fixed unit vector $\boldsymbol{k}, \theta\left(=\theta^{2}\right)$ is the azimuthal angle, and $\boldsymbol{e}_{r}(\theta)$ is a radial unit vector orthogonal 
to the axis of symmetry at azimuth $\theta$. Let $\psi(r)$ be the angle defining the slope of a meridian: $\tan \psi=z^{\prime}(r)$. Then with reference to Section 5, we compute

$$
\boldsymbol{a}_{1}=\boldsymbol{e}_{r}(\theta)+\tan \psi \boldsymbol{k}, \quad \boldsymbol{a}_{2}=r \boldsymbol{e}_{\theta}(\theta),
$$

where $\boldsymbol{e}_{\theta}=\boldsymbol{e}_{r}^{\prime}(\theta)$; the metric and dual metric

$$
\left(a_{\alpha \beta}\right)=\operatorname{diag}\left(\sec ^{2} \psi, r^{2}\right), \quad\left(a^{\alpha \beta}\right)=\operatorname{diag}\left(\cos ^{2} \psi, r^{-2}\right) ;
$$

the curvature

$$
\left(b_{\alpha \beta}\right)=\operatorname{diag}\left(\psi^{\prime} \sec \psi, r \sin \psi\right) ;
$$

the mean and Gaussian curvatures

$$
2 H=r^{-1}(r \sin \psi)^{\prime} \quad \text { and } \quad K=r^{-1} \psi^{\prime} \sin \psi \cos \psi ;
$$

and the curvature cofactor

$$
\left(\tilde{b}^{\alpha \beta}\right)=\operatorname{diag}\left(r^{-1} \sin \psi \cos ^{2} \psi, r^{-2} \psi^{\prime} \cos \psi\right) .
$$

The Laplacian of the mean curvature, needed in (144), is (see (122))

$$
\Delta H=r^{-1} \cos \psi\left[(r \cos \psi) H^{\prime}\right]^{\prime} .
$$

Consider the particular surface of revolution described by

$$
\sin \psi=r(d \ln r+b),
$$

where $b, d$ are constants. Following the procedure outlined in Section 4.3 of [OuYang et al. 1999] and adjusting for differences in notation, with some effort it may be verified that (154) solves the shape equation (144) for a monolayer with a constant spontaneous curvature, provided that

$$
\lambda_{m}=0, \quad p=0 \quad \text { and } \quad d=2 C,
$$

and no tangential distributed force is acting.

In [Ou-Yang et al. 1999] this surface is described in terms of the dimensionless radius

$$
x=r / \bar{r}, \quad \text { where } \quad \bar{r}=\exp (-b / d),
$$

is such that $\sin \psi(\bar{r})=0$, which we use to recast (154) as

$$
\sin \psi=\beta x \ln x, \quad \text { with } \quad \beta=2 C \bar{r} .
$$

Following [Ou-Yang et al. 1999], we fix $\beta<0$ with $|\beta|<e$, corresponding to a negative spontaneous curvature. Evidently, $\sin \psi$ vanishes at $x=0$ and $x=1$ and is maximized at $x=e^{-1}$. Because $\sin \psi \leq 1$ the domain of the variable $x$ is $\left[0, x_{e}\right]$, where

$$
x_{e} \ln x_{e}=|\beta|^{-1},
$$


which yields a unique $x_{e}>1$ [Ou-Yang et al. 1999]. This is the dimensionless equatorial radius, where $\sin \psi=-1$.

To obtain the shape of the surface we integrate $\tan \psi=\varsigma^{\prime}(x)$, where $\varsigma(x)=$ $z(r) / \bar{r}$. Thus,

$$
\varsigma(x)=\int_{x_{e}}^{x} \frac{\beta t \ln t}{\sqrt{1-\beta^{2} t^{2}(\ln t)^{2}}} d t
$$

in which we have chosen the positive root for the cosine and normalized to $\varsigma\left(x_{e}\right)=$ 0 . A numerical quadrature furnishes the upper half of a biconcave discoid, depicted in Figure 3. This is extended by rotational and reflection symmetry to the entire discoid.

Some insight into the mechanics of the system may be gained by computing the transverse shear traction $S$ acting on a parallel of latitude. Assuming the component $\mu$ of the double force to vanish on a parallel, we find, from $(92)_{2}$ and $(127)_{2}$, that $S=\boldsymbol{n} \cdot \boldsymbol{T}^{\alpha} v_{\alpha}$, where

$$
\boldsymbol{n}=\cos \psi \boldsymbol{k}-\sin \psi \boldsymbol{e}_{r} \quad \text { and } \quad \boldsymbol{v}=\cos \psi \boldsymbol{e}_{r}+\sin \psi \boldsymbol{k},
$$

are the surface normal and the normal to a parallel, respectively. Then $(92)_{2}$ and (97) furnish $S=-M_{; \beta}^{\alpha \beta} v_{\alpha}=-\kappa v^{\alpha} H_{, \alpha}$, i.e.,

$$
S=-\kappa \cos \psi H^{\prime}(r),
$$

which may be reduced, using (151), (154) and $(155)_{3}$, to

$$
S=-2 \kappa C r^{-1} \cos \psi \text {. }
$$

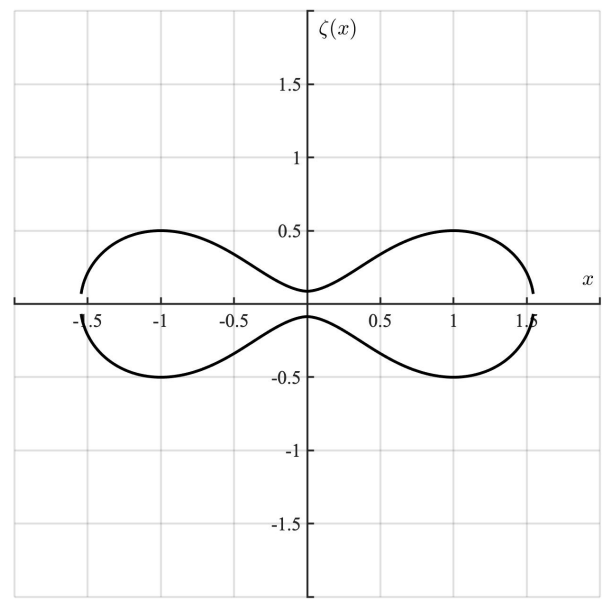

Figure 3. Biconcave discoid $(\beta=-1.4721)$. 
This vanishes at the equator, where $\psi=-\pi / 2$, and therefore meets a necessary condition for reflection symmetry of the surface with respect to the equatorial plane. For, if there were a nonzero shear traction transmitted by the material below the equator to that above, then equilibrium would require that it be balanced by an equal and opposite traction exerted by the part of the membrane above the equator on that below, and this would destroy reflection symmetry. However, the biconcave discoid is not free standing. There is a point force $F \boldsymbol{k}$ acting at the pole, where $\psi=0$, given by

$$
F=-2 \pi \lim _{r \rightarrow 0}(r S)=4 \pi \kappa C,
$$

which was overlooked in [Ou-Yang et al. 1999].

8.3. Mapping a plane cytoskeletal disc to a biconcave discoid. To adapt (154) to the bilayer/cytoskeleton composite, we must select a suitable configuration relative to which the constitutive framework (87) for an isotropic cytoskeleton, say, may be implemented. Because the literature is ambiguous concerning this issue, we consider a plane disc for the sake of illustration, and seek a strain-energy function which is such as to admit (154) as an equilibrium configuration in the absence of any distributed tangential forces acting on the bilayer or cytoskeleton.

We parametrize the disc by the position function $\boldsymbol{\xi}\left(\theta^{\alpha}\right)=\rho(r) \boldsymbol{e}_{r}(\theta)$ (see (6)). The induced tangent basis elements, $\boldsymbol{A}_{\alpha}=\boldsymbol{\xi}_{, \alpha}$, are

$$
\boldsymbol{A}_{1}=\rho^{\prime}(r) \boldsymbol{e}_{r}(\theta) \quad \text { and } \quad \boldsymbol{A}_{2}=\rho(r) \boldsymbol{e}_{\theta}(\theta),
$$

and the metric and dual metric are

$$
\left(A_{\alpha \beta}\right)=\operatorname{diag}\left[\left(\rho^{\prime}\right)^{2}, \rho^{2}\right] \quad \text { and } \quad\left(A^{\alpha \beta}\right)=\operatorname{diag}\left[\left(\rho^{\prime}\right)^{-2}, \rho^{-2}\right] .
$$

With $J_{c}=\sqrt{a / A}$, where $a=\operatorname{det}\left(a_{\alpha \beta}\right)$ and $A=\operatorname{det}\left(A_{\alpha \beta}\right)$, we obtain

$$
J_{c}=r \sec \psi /\left(\rho \rho^{\prime}\right) \text {, }
$$

and

$$
I=a_{\alpha \beta} A^{\alpha \beta}=J_{c}^{2}(\rho / r)^{2}+(r / \rho)^{2} .
$$

Areal incompressibility then yields

$$
I=(\rho / r)^{2}+(r / \rho)^{2},
$$

and furnishes a differential equation for $\rho(r)$ :

$$
\rho \rho^{\prime}=r \sec \psi .
$$

This integrates to

$$
\left(\frac{X}{x}\right)^{2}=\frac{2}{x^{2}} \int_{0}^{x} t \sec \psi(t) d t, \quad \text { where } \quad X=\rho / \bar{r},
$$


and we have imposed $X=0$ at $x=0$ (Figure 4).

The constitutive part of the stress is given by (87). We combine this with (82) (87) and (146) to derive the system

$$
\begin{aligned}
\lambda_{c} \cos ^{2} \psi+2 \varpi^{\prime}(I)\left(\rho^{\prime}\right)^{-2} & =-\kappa C r^{-1} \sin \psi \cos ^{2} \psi+\kappa C^{2} \cos ^{2} \psi, \\
\lambda_{c} r^{-2}+2 \varpi^{\prime}(I) \rho^{-2} & =-\kappa C r^{-2} \psi^{\prime} \cos \psi+\kappa C^{2} r^{-2},
\end{aligned}
$$

which also applies in the case of a comoving cytoskeleton if the multiplier $\lambda_{c}$ is replaced by $\lambda$. Eliminating this multiplier, we obtain

$$
2 \varpi^{\prime}(I)\left[\left(\rho^{\prime}\right)^{-2}-(r / \rho)^{2} \cos ^{2} \psi\right]=-\kappa C \cos ^{2} \psi\left(r^{-1} \sin \psi-\psi^{\prime} \cos \psi\right),
$$

which may be simplified by using (166) to reduce the left-hand side. On the righthand side we use (154), finding that

$$
r^{-1} \sin \psi-\psi^{\prime} \cos \psi=-d .
$$

Then, with (155) we have

$$
\varpi^{\prime}(I)\left[(\rho / r)^{2}-(r / \rho)^{2}\right]=\kappa C^{2},
$$

where, from (168),

$$
\left[(\rho / r)^{2}-(r / \rho)^{2}\right]^{2}=I^{2}-4
$$

With $\cos \psi \in(0,1]$ almost everywhere on the biconcave discoid (Figure 3 ), equation (169) implies that $\rho / r(=X / x)>1$ almost everywhere (Figure 4). Then

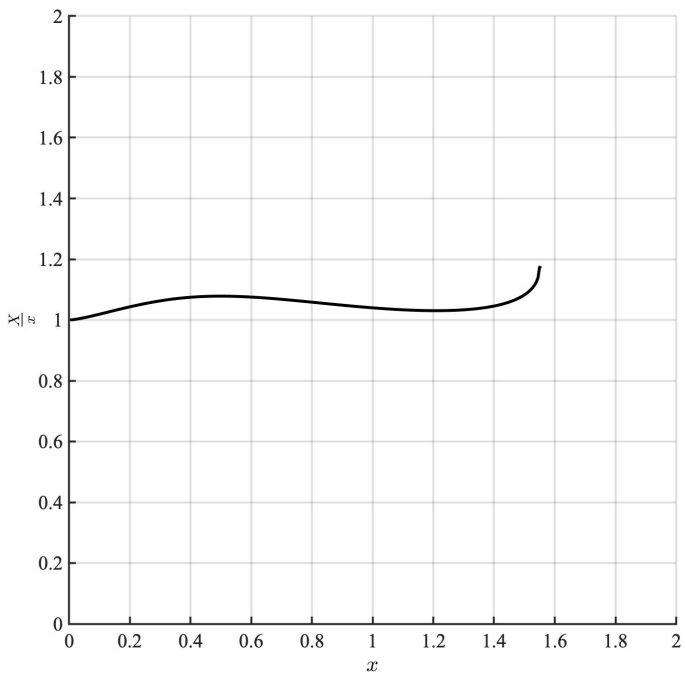

Figure 4. Map from the biconcave discoid to the plane disc $(\beta=-1.4721)$. 
$(\rho / r)^{2}-(r / \rho)^{2}>0$ and (174), (175) deliver

$$
\varpi^{\prime}(I)=\kappa C^{2} / \sqrt{I^{2}-4},
$$

which is meaningful if $I>2$ (as required by (175)) and satisfies (138). Thus,

$$
\varpi(I)=\kappa C^{2} \ln \left[\frac{1}{2}\left(I+\sqrt{I^{2}-4}\right)\right],
$$

normalized to $\varpi(2)=0$.

We are not able to show that (176) satisfies the full Legendre-Hadamard inequality (139). However, as previously noted, the latter is not relevant if the cytoskeleton and bilayer are comoving.

\section{Appendix}

We show that $G(-\boldsymbol{e})=G(\boldsymbol{e})$ if and only if there is a function $S$ such that $G(\boldsymbol{e})=$ $S(\boldsymbol{e} \otimes \boldsymbol{e})$. Sufficiency is immediate. To establish necessity, we show that if $G(-\boldsymbol{e})=$ $G(\boldsymbol{e})$, then $G$ is determined by $\boldsymbol{e} \otimes \boldsymbol{e}$, i.e., that $G(\boldsymbol{a})=G(\boldsymbol{b})$ whenever $\boldsymbol{a} \otimes \boldsymbol{a}=\boldsymbol{b} \otimes \boldsymbol{b}$. The latter yields

$$
a^{2} \boldsymbol{a}=(\boldsymbol{a} \cdot \boldsymbol{b}) \boldsymbol{b} \quad \text { and } \quad b^{2} \boldsymbol{b}=(\boldsymbol{a} \cdot \boldsymbol{b}) \boldsymbol{a},
$$

where $a=|\boldsymbol{a}|$, etc. The combination of these gives $a=b$ and $a^{2} b^{2}=(\boldsymbol{a} \cdot \boldsymbol{b})^{2}$. But there is $\theta \in \mathbb{R}$ such that $\boldsymbol{a} \cdot \boldsymbol{b}=a b \cos \theta$. Thus $\cos \theta= \pm 1$ and either of the two equations yields $\boldsymbol{b}= \pm \boldsymbol{a}$. The first alternative gives $G(\boldsymbol{a})=G(\boldsymbol{b})$; the second yields $G(\boldsymbol{a})=G(-\boldsymbol{b})$, so that if $G$ is insensitive to the choice of sign, as assumed, then $G(\boldsymbol{a})=G(\boldsymbol{b})$ whenever $\boldsymbol{a} \otimes \boldsymbol{a}=\boldsymbol{b} \otimes \boldsymbol{b}$.

\section{Acknowledgments}

Hendrickson gratefully acknowledges support provided by a National Science Foundation Fellowship. Shirani and Steigmann gratefully acknowledge the partial support of the NSF through grant number CMMI-1931064.

\section{References}

[Agrawal and Steigmann 2008] A. Agrawal and D. J. Steigmann, "Coexistent fluid-phase equilibria in biomembranes with bending elasticity”, J. Elasticity 93 (2008), 63-80.

[Agrawal and Steigmann 2009] A. Agrawal and D. J. Steigmann, "Boundary-value problems in the theory of lipid membranes", Contin. Mech. Therm. 21 (2009), 57-82.

[Agrawal and Steigmann 2011] A. Agrawal and D. J. Steigmann, "A model for surface diffusion of trans-membrane proteins on lipid bilayers", Z. Angew. Math. Phys. 62:3 (2011), 549-563.

[Aris 1989] R. Aris, Vectors, tensors and the basic equations of fluid mechanics, Dover Publications, 1989.

[Berdichevsky 2009] V. Berdichevsky, Variational principles of continuum mechanics: part I-fundamentals, Springer, Berlin, 2009. 
[Canham 1970] P. B. Canham, "The minimum energy of bending as a possible explanation of the biconcave shape of the human red blood cell”, J. Theor. Biol. 26:1 (1970), 61-81.

[Ciarlet 2005] P. G. Ciarlet, An introduction to differential geometry with applications to elasticity, Springer, Dordrecht, 2005.

[Cohen and Wang 1984] H. Cohen and C.-C. Wang, "On the response and symmetry of elastic and hyperelastic membrane points", Arch. Ration. Mech. Anal. 85 (1984), 355-391.

[Dharmavaram and Healey 2015] S. Dharmavaram and T. J. Healey, "On the equivalence of local and global area-constraint formulations for lipid bilayer vesicles", Z. Angew. Math. Phys. 66 (2015), 2843-2854. arXiv

[Evans and Skalak 1980] E. Evans and R. Skalak, Mechanics and thermodynamics of biomembranes, CRC Press, Boca Raton, 1980.

[Guven et al. 2019] J. Guven, M. M. Müller, and P. Vázquez-Montejo, "Isometric bending requires local constraints on free edges”, Math. Mech. Solids 24:12 (2019), 4051-4077. arXiv

[Helfrich 1973] W. Helfrich, "Elastic properties of lipid bilayers: theory and possible experiments", Z. Naturforsch. 28 (1973), 693-703.

[Herant and Dembo 2006] M. Herant and M. Dembo, "Active cellular protrusion: continuum theories and models", pp. 204-224 in Cytoskeletal mechanics: models and measurements in cell mechanics, edited by M. R. K. Mofrad and R. D. Kamm, Cambridge University Press, Cambridge, 2006.

[Hilgers and Pipkin 1993] M. G. Hilgers and A. C. Pipkin, "Energy-minimizing deformations of elastic sheets with bending stiffness", J. Elasticity 31 (1993), 125-139.

[Jenkins 1977] J. T. Jenkins, "The equations of mechanical equilibrium of a model membrane", SIAM J. Appl. Math. 32:4 (1977), 755-764.

[Kamm and Mofrad 2006] R. D. Kamm and M. R. K. Mofrad, "Introduction, with the biological basis for cell mechanics", pp. 1-17 in Cytoskeletal mechanics: models and measurements in cell mechanics, edited by M. R. K. Mofrad and R. D. Kamm, Cambridge University Press, Cambridge, 2006.

[Krishnaswamy 1996] S. Krishnaswamy, "A cosserat-type model for the red blood cell wall”, Int. J. Eng. Sci. 34:8 (1996), 873-899.

[Murdoch and Cohen 1979] A. I. Murdoch and H. Cohen, "Symmetry considerations for material surfaces", Arch. Ration. Mech. Anal. 72 (1979), 61-98.

[Naghdi 1972] P. M. Naghdi, "The theory of shells and plates”, in S. Flügge's Handbuch der Physik, vol. VIa/2, edited by C. Truesdell, Springer, Berlin, 1972.

[Nitsche 1993] J. C. C. Nitsche, "Boundary value problems for variational integrals involving surface curvatures”, Quart. Appl. Math. 51:2 (1993), 363-387.

[Noll 1958] W. Noll, "A mathematical theory of the mechanical behavior of continuous media", Arch. Ration. Mech. Anal. 2 (1958), 197-226.

[Noll 2004] W. Noll, "On material frame-indifference", Five contributions to natural philosophy, 2004, Available at http://www.math.cmu.edu/ wn0g/noll/FC.pdf.

[Ou-Yang et al. 1999] Z.-C. Ou-Yang, J.-X. Liu, and Y.-Z. Xia, Geometric methods in the elastic theory of membranes in liquid crystal phases, World Scientific, Singapore, 1999.

[Pan et al. 2018] L. Pan, R. Yan, W. Li, and K. Xu, "Super-resolution microscopy reveals the native ultrastructure of the erythrocyte cytoskeleton”, Cell Rep. 22:5 (2018), 1151-1158.

[Rosso and Virga 1999] R. Rosso and E. G. Virga, "Adhesive borders of lipid membranes", Proc. R. Soc. Lond. A 455:1992 (1999), 4145-4168. 
[Scriven 1960] L. E. Scriven, "Dynamics of a fluid interface: equation of motion for Newtonian surface fluids", Chem. Eng. Sci. 12:2 (1960), 98-108.

[Stamenović 2006] D. Stamenović, "Models of cytoskeletal mechanics based on tensegrity", pp. 103-128 in Cytoskeletal mechanics: models and measurements in cell mechanics, edited by M. R. K. Mofrad and R. D. Kamm, Cambridge University Press, Cambridge, 2006.

[Steigmann 1990] D. J. Steigmann, “Tension-field theory”, Proc. R. Soc. Lond. A 429:1876 (1990), 141-173.

[Steigmann 2003] D. J. Steigmann, "Irreducible function bases for simple fluids and liquid crystal films", Z. angew. Math. Phys. 54 (2003), 462-477.

[Steigmann 2010] D. J. Steigmann, "Applications of polyconvexity and strong ellipticity to nonlinear elasticity and elastic plate theory", pp. 265-299 in Poly-, quasi- and rank-one convexity in applied mechanics, vol. 516, edited by J. Schröder and P. Neff, Springer, Vienna, 2010.

[Steigmann 2018] D. J. Steigmann, "Continuum theory for elastic sheets formed by inextensible crossed elasticae", Int. J. Non-Linear Mech. 106 (2018), 324-329.

[Tartibi et al. 2015] M. Tartibi, Y. X. Liub, G.-Y. Liub, and K. Komvopoulos, "Single-cell mechanics - an experimental-computational method for quantifying the membrane-cytoskeleton elasticity of cells", Acta Biomater. 27 (2015), 224-235.

[Toupin 1962] R. A. Toupin, "Elastic materials with couple-stresses", Arch. Ration. Mech. Anal. 11 (1962), 385-414.

[Virga 1994] E. G. Virga, Variational theories for liquid crystals, Chapman \& Hall, London, 1994.

[Wang and Pipkin 1986] W.-B. Wang and A. C. Pipkin, "Inextensible networks with bending stiffness", Quart. J. Mech. Appl. Math. 39:3 (1986), 343-359.

[Zheng 2003] Q.-S. Zheng, "Irreducible function bases for simple fluids and liquid crystal films: a new derivation", Z. angew. Math. Phys. 54 (2003), 478-483.

[Zheng et al. 1992] Q.-S. Zheng, J. Betten, and A. J. M. Spencer, "The formulation of constitutive equations for fibre-reinforced composites in plane problems: part I", Arch. Appl. Mech. 62 (1992), $530-543$.

Received 24 Oct 2019. Revised 27 Feb 2020. Accepted 4 Apr 2020.

BRETT HENDRICKSON: bretth@berkeley .edu

Department of Mechanical Engineering, University of California, Berkeley, 6112 Etcheverry Hall, Berkeley, CA 94720-1740, United States

MiLAD SHIRANI: milad_shirani@berkeley.edu

Department of Mechanical Engineering, University of California, Berkeley, 6112 Etcheverry Hall, Berkeley, CA 94720-1740, United States

DAVID J. STEIGMANN: dsteigmann@berkeley.edu

Department of Mechanical Engineering, University of California, Berkeley, 6133 Etcheverry Hall, Berkeley, CA 94720-1740, United States 
EDITORIAL BOARD

ANTONIO CARCATERRA

ERIC A. CARLEN

FRANCESCO DELL'ISOLA

RAFFAELE ESPOSITO

ALBERT FANNJIANG

Gilles A. FranCFORT

PiERANGELO MARCATI

JEAN-JACQUES MARIGO

PETER A. MARKOWICH

MARTIN OSTOJA-STARZEWSKI

PIERRE SEPPECHER

DAVID J. STEIGMANN

PAUl STEINMANN

PierRe M. SuQueT

MANAGING EDITORS

MICOL AMAR

EMILIO BARCHIESI

ANGELA MADEO

MARTIN OSTOJA-STARZEWSKI

ADVISORY BOARD

ADNAN AKAY

Holm AltenbaCH

MICOL AMAR

HARM ASKES

TEODOR ATANACKOVIĆ

VICTOR BERDICHEVSKY

GUY BOUCHITTÉ

ANDREA BRAIDES

ROBERTO CAMASSA

MAURO CARFORE

ERIC DARVE

FELIX DARVE

ANNA DE MASI

Gianpietro Del Piero

EMMANUELE Di BENEDETTO

VICTOR A. EREMEYEV

BERNOLD FIEDLER

IRENE M. GAMBA

DAVID Y. GAO

Sergey GaVRILyuk

Timothy J. HEALEY

DOMINIQUe JEULiN

ROGER E. KHAYAT

Corrado LATTANZIO

ROBERT P. LIPTON

ANGELO LUONGO

ANGELA MADEO

JUAN J. MANFREDI

CARLO MARCHIORO ANIL MisRa

ROBERTO NATALINI PATRIZIO NEFF

Thomas J. PENCE

ANDREY PIATNITSKI

ERRICO PRESUTTI

MARIO Pulvirenti

LUCIO RUSSO

Miguel A. F. SAnjuaN

PATRICK SElVAdurai

MiroslaV ŠILHAVÝ

GUIDO SWEERS

ANTOINETTE TORDESILLAS

LEV TRUSKINOVSKY

JUAN J. L. VELÁZQUEZ

VINCENZO VESPRI

ANGELO VULPIANI msp.org/memocs

Università di Roma "La Sapienza", Italia

Rutgers University, USA

(CO-CHAIR) Università di Roma "La Sapienza", Italia

(TREASURER) Università dell'Aquila, Italia

University of California at Davis, USA

(CO-CHAIR) Université Paris-Nord, France

Università dell' Aquila, Italy

École Polytechnique, France

DAMTP Cambridge, UK, and University of Vienna, Austria

(CHAIR MANAGING EDITOR) Univ. of Illinois at Urbana-Champaign, USA

Université du Sud Toulon-Var, France

University of California at Berkeley, USA

Universität Erlangen-Nürnberg, Germany

LMA CNRS Marseille, France

Università di Roma "La Sapienza”, Italia

Università degli Studi dell'Aquila, Italy

Université de Lyon-INSA (Institut National des Sciences Appliquées), France

(CHAIR MANAGING EDITOR) Univ. of Illinois at Urbana-Champaign, USA

Carnegie Mellon University, USA, and Bilkent University, Turkey

Otto-von-Guericke-Universität Magdeburg, Germany

Università di Roma "La Sapienza", Italia

University of Sheffield, UK

University of Novi Sad, Serbia

Wayne State University, USA

Université du Sud Toulon-Var, France

Università di Roma Tor Vergata, Italia

University of North Carolina at Chapel Hill, USA

Università di Pavia, Italia

Stanford University, USA

Institut Polytechnique de Grenoble, France

Università dell'Aquila, Italia

Università di Ferrara and International Research Center MEMOCS, Italia

Vanderbilt University, USA

Gdansk University of Technology, Poland

Freie Universität Berlin, Germany

University of Texas at Austin, USA

Federation University and Australian National University, Australia

Université Aix-Marseille, France

Cornell University, USA

École des Mines, France

University of Western Ontario, Canada

Università dell' Aquila, Italy

Louisiana State University, USA

Università dell'Aquila, Italia

Université de Lyon-INSA (Institut National des Sciences Appliquées), France

University of Pittsburgh, USA

Università di Roma "La Sapienza", Italia

University of Kansas, USA

Istituto per le Applicazioni del Calcolo "M. Picone", Italy

Universität Duisburg-Essen, Germany

Michigan State University, USA

Narvik University College, Norway, Russia

Università di Roma Tor Vergata, Italy

Università di Roma "La Sapienza", Italia

Università di Roma "Tor Vergata", Italia

Universidad Rey Juan Carlos, Madrid, Spain

McGill University, Canada

Academy of Sciences of the Czech Republic

Universität zu Köln, Germany

University of Melbourne, Australia

École Polytechnique, France

Bonn University, Germany

Università di Firenze, Italia

Università di Roma La Sapienza, Italia

MEMOCS (ISSN 2325-3444 electronic, 2326-7186 printed) is a journal of the International Research Center for the Mathematics and Mechanics of Complex Systems at the Università dell'Aquila, Italy.

Cover image: "Tangle” by @ John Horigan; produced using the Context Free program (contextfreeart.org).

\section{PUBLISHED BY}

\section{I. mathematical sciences publishers} nonprofit scientific publishing http://msp.org/ 
Mathematics and Mechanics of Complex Systems vol. 8 no. 1

Second-order work criterion and divergence criterion: a full 1 equivalence for kinematically constrained systems

Jean Lerbet, Noël Challamel, François Nicot and Felix

Darve

On the generalization of the Brewster Law

Fabrizio Davì

IBVP for electromagneto-elastic materials: variational approach

Amirhossein Amiri-Hezaveh, Pouyan Karimi and

Martin Ostoja-Starzewski

Equilibrium theory for a lipid bilayer with a conforming cytoskeletal membrane

Brett Hendrickson, Milad Shirani and David J.

Steigmann

MEMOCS is a journal of the International Research Center for the Mathematics and Mechanics of Complex Systems at the Università dell' Aquila, Italy.

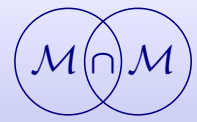

